\title{
Non-Gaussianity as a Signature of a Quantum Theory of Gravity
}

\author{
Richard Howlø, ${ }^{1,2,3,{ }^{*}}$ Vlatko Vedral,,${ }^{4,5}$ Devang Naik, ${ }^{6}$ Marios Christodoulou $\odot,{ }^{2,4}$ Carlo Rovelli, ${ }^{7,8,9}$ \\ and Aditya Iyer ${ }^{4}$ \\ ${ }^{1}$ School of Mathematical Sciences, University of Nottingham, University Park, Nottingham, NG7 2RD, United \\ Kingdom \\ ${ }^{2}$ QICI Quantum Information and Computation Initiative, Department of Computer Science, The University of \\ Hong Kong, Pokfulam Road, Hong Kong \\ ${ }^{3}$ Quantum Group, Department of Computer Science, University of Oxford, Wolfson Building, Parks Road, Oxford, \\ OX1 3QD, United Kingdom \\ ${ }^{4}$ Clarendon Laboratory, Department of Physics, University of Oxford, Oxford, OX1 3PU, United Kingdom \\ ${ }^{5}$ Centre for Quantum Technologies, National University of Singapore, Block S15, 3 Science Drive 2, \\ Singapore 117543 \\ ${ }^{6}$ LP2N, Laboratoire Photonique, Numérique et Nanosciences, Université Bordeaux-IOGS-CNRS:UMR 5298, \\ 33400 Talence, France \\ ${ }^{7}$ CPT, Aix-Marseille Université, Université de Toulon, CNRS, F-13288 Marseille, France \\ ${ }^{8}$ Perimeter Institute, 31 Caroline Street North, Waterloo, Ontario, N2L 2Y5, Canada \\ ${ }^{9}$ The Rotman Institute of Philosophy, Western University, 1151 Richmond Street North, London, Ontario, N6A5B7, \\ Canada
}

(Received 30 April 2020; accepted 21 January 2021; published 17 February 2021)

\begin{abstract}
Tabletop tests of quantum gravity (QG) have long been thought to be practically impossible. However, remarkably, because of rapid progress in quantum information science (QIS), such tests may soon be achievable. Here we uncover an exciting new theoretical link between QG and QIS that also leads to a radical new way of testing QG with QIS experiments. Specifically, we find that only a quantum, not classical, theory of gravity can create non-Gaussianity, a QIS resource that is necessary for universal quantum computation, in the quantum field state of matter. This allows tests based on QIS in which nonGaussianity in matter is used as a signature of QG. In comparison with previous studies testing QG with QIS where entanglement is used to witness QG when all other quantum interactions are excluded, our non-Gaussianity witness cannot be created by direct classical gravity interactions, facilitating tests that are not constrained by the existence of such processes. Our new signature of QG also enables tests that are based on just a single quantum system rather than a multipartite quantum system, simplifying previously considered experimental setups. We describe a tabletop test of QG that uses our non-Gaussianity signature and that is based on just a single quantum system, a Bose-Einstein condensate, in a single location. In contrast to proposals based on optomechanical setups, Bose-Einstein condensates have already been manipulated into massive nonclassical states, aiding the prospect of testing QG soon.
\end{abstract}

DOI: 10.1103/PRXQuantum.2.010325

\section{INTRODUCTION}

Shortly after Einstein formulated general relativity (GR), he wondered how quantum theory (QT) would modify it [1]. Yet, more than 100 years later, there is still no consensus on how these two fundamental theories should

\footnotetext{
*rjhowl@gmail.com
}

Published by the American Physical Society under the terms of the Creative Commons Attribution 4.0 International license. Further distribution of this work must maintain attribution to the author(s) and the published article's title, journal citation, and DOI. be unified [2-6]. The conventional approach is to apply the principles of QT to gravity [7], resulting in a quantum gravity $(\mathrm{QG})$ theory, such as string theory [8-11] or loop QG [12-14]. However, since it is not as straightforward to apply QT to gravity as compared with the other fundamental forces $[15,16]$, an alternative class of unifying theories has been developed, classical gravity (CG) theories, such as semiclassical gravity [15,17-19], where matter is quantized but gravity remains fundamentally classical [16].

The hope has been that theoretical study alone would allow us to discover how GR and QT are unified in nature. However, the fact that there are several proposals 
illustrates that this is unlikely to happen and that experimental intervention is required [16]. Until recently, the common view was that there is little hope of laboratory tests of QG since we need to probe GR near a small length scale, the Planck length, where QT effects of spacetime become relevant [20], but for which we would likely need to build a Milky Way-sized particle accelerator [20,21]. However, there is another important scale, the Planck mass scale, where gravitational effects of massive quantum systems become relevant, allowing us, in particular, to distinguish QG from CG [22]. This mass scale should be within reach soon in laboratory settings due to the rapidly developing field of quantum information science (QIS) [23,24]. This has led to several proposals being developed recently for tests of QG using techniques of QIS [23,25-33]. Of these, a particularly promising experimental proposal is the Bose-Marletto-Vedral (BMV) experiment [27-30], where, under the condition that all other quantum interactions can be excluded, the creation of entanglement between two microspheres, each in a superposition of two locations, is used as a witness of QG. Because of the strength of this effect, and the hope of mesoscopic superposition states in optomechanical systems [34,35], it is thought that this QIS-inspired experimental test of QG could be possible soon [27-30].

An issue with an entanglement-based test of QG, however, is that classical, as well as quantum, theories of gravity can create entanglement. For example, modes of a quantum field can become entangled by a classically expanding universe [36-43]. This has resulted in questions concerning the reliability of using entanglement as a witness of QG [44-47]. In particular, in the BMV proposal, entanglement as a witness of QG is based on the assumption that CG acts as a local operations and communication channel (LOCC) $[27,28]$ or, more generally, as a local classical-information mediator [29,30,47], which can never create entanglement. However, it is in theory possible that CG could cause two spatially separated quantum matter systems to directly couple with one another, invalidating the LOCC and classical-information mediator arguments and leading to entanglement generation in experiments [27-30]. For instance, such direct CG interactions could be due to nonlocal effects associated with CG [27-30], or quasilocal CG effects, such as tunneling between two quantum matter systems, which we consider in Sec. IV B.

Here we take a radically different approach to testing QG with QIS. Rather than concentrating on how QG can act as a quantum-information mediator in comparison with a classical communication channel $[27,28]$ or a classicalinformation mediator $[29,30]$, we consider how just the simple process of adding a "hat" to classical gravitational degrees of freedom (i.e., turning them into quantum operators) results in a theory that, in contrast to its classical counterpart, can create non-Gaussianity in the quantum (a)

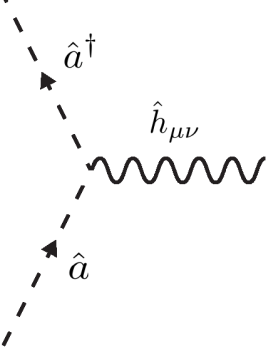

(b)

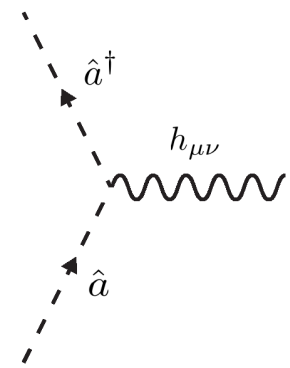

FIG. 1. (a) Basic Feynman diagram for matter interacting with QG where matter emits a graviton, which is associated with $\hat{h}_{\mu \nu}$. For simplicity, we represent matter by a real scalar field such that $\hat{a}^{\dagger}$ and $\hat{a}$ are the creation and annihilation operators of matter. The interaction is then associated with three quantum operators and, therefore, can induce non-Gaussianity. (b) We can illustrate the analogous interaction between matter and classical gravity with a similar diagram except that now the gravitational leg represents a classical gravitational wave $h_{\mu \nu}$ rather than a graviton. Since this CG interaction is associated with just two quantum operators $\hat{a}^{\dagger}$ and $\hat{a}$, it cannot, in contrast to the QG interaction, induce non-Gaussianity. Although these diagrams represent weak-field, perturbative gravitational interactions, the fact that CG cannot create non-Gaussianity also applies to the strong-field, nonperturbative regime of gravity, as shown in Sec. II.

field of matter. Non-Gaussianity is a key resource in continuous-variable QIS (CVQIS), where quantum information is encoded in degrees of freedom with a continuous spectrum. For example, it is necessary in order to perform universal quantum computation [48,49], and the reason that, in the exclusion of all other quantum interactions, only a quantum rather than a classical theory of gravity can create non-Gaussianity in the quantum field state of matter is for the same reason that non-Guassianity is required for universal quantum computation: non-Gaussianity is created by processes that are nonquadratic in quantum operators [50-53], and only QG, compared with CG, can contain such processes. Although our argument applies to both the weak-field regime and the strong-field regime of gravity, it is perhaps most intuitively understood from a perturbative weak-field perspective. In this case, the simplest interaction between matter and gravity in QG is where matter creates a graviton. As illustrated in Fig. 1, the corresponding Feynman diagram contains three quantum operators and, therefore, induces non-Gaussianity. On the other hand, in CG, we would remove the "hat" of the gravitational degrees of freedom, leaving a quadratic Hamiltonian, which preserves Gaussianity [54].

In comparison to entanglement, since our non-Gaussianity indicator is not based on LOCC or classical-information mediator arguments, this indicator of QG is not reliant on the nonexistence of direct CG interactions, which we illustrate in Sec. IV B. Therefore, as 
long as we are working in an experimental situation where nongravitational quantum interactions can be ignored (just as in tests based on entanglement [27-30]) and where matter can be well represented by a quantum field, nonGaussianity can be used as a signature of QG in experimental tests without the need for the additional assumption of there being no direct CG interactions [27-30]. A further advantage of a non-Gaussianity signature is that a single system can be non-Gaussian, allowing tests of QG that are based on just a single system rather than a multipartite system. We illustrate this with a tabletop test of QG that uses a single Bose-Einstein condensate (BEC) in a single location.

In addition to being just a single quantum system without any spatial superposition, our experimental proposal also uses a type of quantum technology (BECs) for which certain massive quantum states have already been created [55-59]. This is in contrast to proposals based on optomechanical setups, where massive nonclassical states have yet to be generated. BECs also offer a contrasting method to distinguish the QG signal from electromagnetic noise. As with previous proposals, such as the BMV proposal, it is vital that we can attribute the sought QG effect from the analogous effect that is generated through electromagnetic interactions. For the BMV proposal this effect is entanglement, which electromagnetic as well as gravitational interactions will naturally generate, whereas in the test proposed here this is non-Gaussianity, which electromagnetic interactions would also naturally generate since they are fundamentally quantum interactions.

To isolate the gravitational non-Gaussian signal from an electromagnetic one, we use the fact that only the electromagnetic interaction can be screened and, in particular, that BECs generically have Feshbach resonances. Electromagnetism can be screened since it has both positive and negative charges, whereas gravity is universal, coupling to all forms of energy in the same way. In BECs, this is immediately apparent since the atoms have zero overall electromagnetic charge, resulting in them naturally interacting only through van der Waals interactions and, in most cases, magnetic dipole-dipole interactions (MDDIs) at very low temperatures. This allows the use of an extraordinary property of BEC and cold atom experiments to distinguish the electromagnetic and gravitational effects. This property is the presence of optical and magnetic Feshbach resonances, which are used in BEC experiments to control the strength of the electromagnetic interactions between the atoms by the application of an external magnetic or optical field. This has become a vital tool of BEC experiments that has facilitated numerous explorations of fundamental physics [60,61].

By applying a magnetic or optical field to the BEC, we can in principle set the overall strength of the electromagnetic interactions to zero without affecting the strength of the gravitational interactions $[60,61]$. Then a non-Gaussian signal can be attributed to only gravitational interactions. This method contrasts with that used in optomechanical proposals, where the distance between micro-objects is increased to a level where gravitational interactions are greater than the electromagnetic van der Waals interactions. In that case, both the electromagnetic interactions and the gravitational interactions are suppressed by increasing the distance, whereas applying an external magnetic or optical field to a BEC affects only the former.

\section{NON-GAUSSIANITY AS A SIGNATURE OF QUANTUM GRAVITY}

Consider a free, real scalar quantum field. The Hamiltonian of this system can be written as a collection of quantum simple harmonic oscillators: $\hat{H}=\sum_{k} \hbar \omega_{k}\left[\hat{a}_{k}^{\dagger} \hat{a}_{k}+\right.$ $1 / 2]$, where $\hat{a}_{k}^{\dagger}$ and $\hat{a}_{k}$ are creation and annihilation operators of mode $k, \omega_{k}$ is the angular frequency, and we assume a discrete mode spectrum for simplicity [62]. For each oscillator we can associate positionlike and momentumlike operators, $\hat{x}_{k}:=\hat{a}_{k}+\hat{a}_{k}^{\dagger}$ and $\hat{p}_{k}:=i\left(\hat{a}_{k}^{\dagger}-\hat{a}\right)$, known as quadrature operators, which are observables, with a continuous eigenspectrum: $\hat{x}_{k}|x\rangle_{k}=x_{k}|x\rangle_{k}$ and $\hat{p}_{k}|p\rangle_{k}=$ $p_{k}|p\rangle_{k}$. The quadrature eigenvalues, $x_{k}$ and $p_{k}$, can be used as continuous variables to describe the entire quantum field system, and we can view this as a continuous phase space on which we encode our quantum information [54]. This approach to encoding quantum information can also be straightforwardly extended to general bosonic and fermionic quantum fields [63-66].

Rather than describing this system using a density operator $\hat{\rho}$, an equivalent representation is provided by the Wigner function [67]. This is a quasi-probabilitydistribution defined over phase space, analogous to probability distributions used in classical statistical mechanics. For example, for a single mode, the Wigner function can be obtained through [68]

$$
W_{\hat{\rho}}(x, p)=\frac{1}{2 \pi} \int d y e^{-i y p}\langle x+y|\hat{\rho}| x-y\rangle .
$$

$W_{\hat{\rho}}(x, p)$ is a quasi-probability-distribution since, although it takes on real values and is normalized to unity, it can also take on negative values. The states for which the Wigner function takes on negative values, therefore, have no classical counterpart, and are considered to be highly nonclassical states [69].

The only states that have negative Wigner functions are non-Gaussian states, such as Fock states or Schrödinger cat states [70]. Gaussian states, on the other hand, such as coherent states, squeezed states, and thermal states, have only positive Wigner functions [54,71]. Here a Gaussian state of a quantum field is defined as one for which its Wigner function is a Gaussian distribution [54]. Such a state is fully characterized by the first and second moments 
of the quadrature operators or, equivalently, by the onepoint and two-point correlation functions of the quantum field $[50,52,54]$.

The classification of Gaussian and non-Gaussian states is very important in CVQIS. For example, universal quantum computation with pure states is possible only with non-Gaussian states or operations $[48,49]$, while Gaussian states and operations can be efficiently simulated on a classical computer [72-75]. Furthermore, non-Gaussian states or operations are required for violation of Bell inequalities [76-83]. These and additional examples, such as implementing entanglement distillation [75], have led to non-Gaussianity being classified as a CVQIS resource for which measures and witnesses have been derived [84-93], just as for entanglement.

Given the significance of Gaussian and non-Gaussian states in CVQIS, it is important to distinguish the type of Hamiltonians that can create such states: a Hamiltonian that is at most quadratic in quadratures, or equivalently in annihilation and creation operators, can only ever map a Gaussian state to another Gaussian state [65,94-98]. That is, the Hamiltonian must be of the form

$$
\hat{H}=\sum_{k} \boldsymbol{\lambda}_{k}(t) \hat{\boldsymbol{x}}_{k}+\sum_{k, l} \hat{\boldsymbol{x}}_{k}^{T} \boldsymbol{\mu}_{k l}(t) \hat{\boldsymbol{x}}_{l}
$$

where $\hat{\boldsymbol{x}}_{k}^{T}:=\left(\hat{x}_{k}, \hat{p}_{k}\right)$, and $\boldsymbol{\lambda}_{k}(t)$ and $\boldsymbol{\mu}_{k l}(t)$ are $2 \times 1$ and $2 \times 2$ real-valued matrices of arbitrary functions of time. Although we assume a discrete, finite mode spectrum here for simplicity, the extension to infinite and continuous modes is straightforward [50-53].

The Hamiltonian (2) preserves Gaussianity since it is associated with a general Bogoliubov transformation, which is a linear transformation of the quadratures (and, therefore, phase space) that preserves their commutation relations [54]. Any other Hamiltonian (i.e., one that is not linear or quadratic in quantum operators) will in general create non-Gaussianity $[48,49,54]$.

A free quantum field has a Hamiltonian that is of the form of Eq. (2) since it contains only the kinetic and mass terms, and so is necessarily quadratic in the field. For example, the free Hamiltonian for a real scalar quantum field $\hat{\phi}$ is [62]

$$
\hat{H}=\frac{1}{2} \int d^{3} \boldsymbol{r}\left[\left(\partial_{t} \hat{\phi}\right)^{2}+(\nabla \hat{\phi})^{2}+m^{2} \hat{\phi}^{2}\right],
$$

where $m$ is the mass of the field. Expanding the field in annihilation and creation operators $\hat{\phi}=\sum_{k}\left[u_{k}(t) \hat{a}_{k}+\right.$ $\left.v(t) \hat{a}_{k}^{\dagger}\right]$ results in a Hamiltonian of the form of Eq. (2) [62].

Now consider interacting this quantum field with a classical entity $\mathcal{G}$, which could depend on space and time. If we take the classical interaction to not induce quantum self-interactions of $\hat{\phi}$, then $\mathcal{G}$ and $\hat{\phi}$ can interact only through Hamiltonian terms that are linear or quadratic in $\hat{\phi}$ [99]. For example, the classical interaction could occur through a Hamiltonian term such as $(\nabla \hat{\phi})^{2} f[\mathcal{G}]$, where $f$ is a real functional of $\mathcal{G}$. Then expanding $\hat{\phi}$ in annihilation and creation operators, we would still find a Hamiltonian that is of the form of Eq. (2), with $\mathcal{G}$ just absorbed into the time-dependent coupling constants. That is, the Hamiltonian of the classical interaction preserves Gaussianity, and this would apply to a classical interaction with any type of quantum field, not just a real scalar field $\hat{\phi}$.

In contrast, if we quantize $\mathcal{G}$ such that we interact $\hat{\phi}$ (or any other type of quantum field) with a quantum entity, then it is possible for the resulting Hamiltonian to be of higher order than quadratic in quantum operators, and thus induce non-Gaussianity. Therefore, any sign of the creation of non-Gaussianity in the state of a quantum field would be evidence of a quantum interaction.

Because of the universal coupling of gravity, we can apply this argument to determine whether gravity obeys a quantum or classical theory. In this case, if we are working in a situation where all other quantum interactions can be ignored, the matter Hamiltonian contains only the kinetic and mass terms of the matter quantum field, to which gravity couples. If there were terms that were neither linear nor quadratic in the quantum matter field, and which thus induce quantum self-interactions of matter, then these would have to be associated with a nongravitational force since these terms must also be present in flat space. Therefore, as we are assuming a situation where all interactions other than gravity can be ignored, these terms are not present.

For example, if, for simplicity, we used a real scalar field $\hat{\phi}$ to describe matter and ignored a possible quadratic Ricci scalar coupling term [100], then the Hamiltonian of CG would be given by Eq. (3) but with $\sqrt{\mathfrak{g}}$ multiplying each term, where $\mathfrak{g}$ is the determinant of the spatial metric tensor [14,101-103]. This Hamiltonian would preserve Gaussianity. In contrast, in QG there must be an operator associated with the gravitational field, which would result in Gaussianity no longer being preserved. For instance, in loop QG, $\mathfrak{g}$ would be quantized in this example [14,101-103], and in linearized QG, we would perturb the gravitational metric around a classical spacetime background metric and quantize only the perturbation [104-107]. Similarly, in the nonrelativistic Newtonian limit, only the temporal component of the perturbed metric would be used, which is quantized and associated with the Newtonian gravitational potential $\Phi[108]$.

In summary, creation of non-Gaussianity would provide evidence for a quantum theory of gravity. Since all known fundamental interactions with matter, such as electromagnetism, have interaction Hamiltonians with terms that are quadratic in matter fields [62,102], non-Gaussianity could also be used to evidence that these are indeed quantum interactions [109]. 
(a)

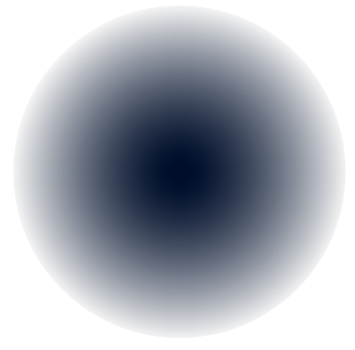

(b)

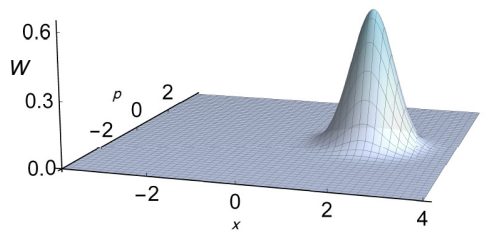

(c)

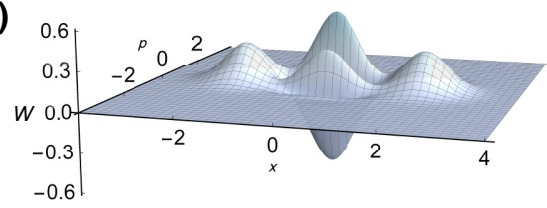

FIG. 2. Illustration of the proposal for a tabletop test of QG. (a) A single spherical BEC of $10^{9}$ atoms and radius $R=200 \mu \mathrm{m}$ is left to self-interact gravitationally for around $t=2 \mathrm{~s}$. If QG acts, non-Gaussianity is induced in its quantum state, whereas if CG acts, Gaussianity is preserved. Each atom is equally delocalized across the extent of the BEC but, since the BEC is in a spherical harmonic trap, the density of the BEC is greatest at its center and drops off to zero asymptomatically, as illustrated. The BEC is initially in a Gaussian state, and its Wigner function $W$ is displayed in (b). Here, for simplicity, a coherent state $|\alpha\rangle$ is assumed, but this can also be squeezed to increase the signalto-noise ratio, as discussed in the main text. If QG acts, and the interaction time were long enough, then gravity could even force the coherent state to a Yurke-Stoler cat state $(|\alpha\rangle+i|-\alpha\rangle) / \sqrt{2}$. The Wigner function for such a non-Gaussian state is illustrated in (c). If, however, CG acts, then the state will remain Gaussian. In the nonrelativistic CG limit, the state will remain a coherent state, whereas relativistic CG effects would, in principle, squeeze the state but keep it Gaussian. In practice, the interaction time would not be long enough for such a dramatic effect as a coherent state changing to a Yurke-Stoler state, and instead smaller deviations from a Gaussian distribution are sought (see the main text). $\alpha=2$ is used in the plots, whereas in practice the BEC will have an amplitude of around $|\alpha|=10^{4.5}$, the square root of the number of atoms.

\section{TESTING QUANTUM GRAVITY WITH A SINGLE QUANTUM SYSTEM}

We now consider a tabletop test of QG that uses our nonGaussianity witness (Fig. 2). This experiment is based on a single BEC that is in a single location, and is an experiment to which an entanglement witness of QG could not be applied.

\section{A. Non-Gaussianity as a signature of quantum gravity in a Bose-Einstein condensate}

A Bose gas can be described by a nonrelativistic scalar quantum field $\hat{\Psi}(\boldsymbol{r})$, which creates an atom at position $\boldsymbol{r}$ [61]. Assuming that we are working at low enough temperatures such that the ground state is macroscopically occupied, we ignore the thermal component of the gas and take $\hat{\Psi}(\boldsymbol{r}) \approx \psi(\boldsymbol{r}) \hat{a}$, where $\psi(\boldsymbol{r})$ is the wave function of a condensed atom and $\hat{a}$ is the annihilation operator for the condensate [61]. The identical atoms are then all in the same state, have the same wave function, and are equally delocalized across the BEC.

These atoms will interact gravitationally with each other, and since this is a nonrelativistic system, it is appropriate to take the nonrelativistic (Newtonian) limit of gravity, which all gravitational theories must contain. The fully classical interaction Hamiltonian for Newtonian gravity is

$$
H_{\mathrm{int}}=\frac{1}{2} \int d^{3} \boldsymbol{r} \rho(\boldsymbol{r}) \Phi(\boldsymbol{r}),
$$

where $\Phi(\boldsymbol{r})$ is the classical Newtonian potential and $\rho(\boldsymbol{r})$ is the classical mass density. If gravity obeys quantum theory, then we must quantize both $\rho(\boldsymbol{r})$ and $\Phi(\boldsymbol{r})$, whereas if we have CG, then we quantize only the former. Since $\hat{\rho}(\boldsymbol{r})=$ $m \hat{\Psi}^{\dagger}(\boldsymbol{r}) \hat{\Psi}^{\dagger}(\boldsymbol{r})$ for a BEC, this results in the respective QG and $\mathrm{CG}$ interaction Hamiltonians

$$
\begin{aligned}
& \hat{H}_{\mathrm{QG}}=\frac{1}{2} m \int d^{3} \boldsymbol{r}: \hat{\Psi}^{\dagger}(\boldsymbol{r}) \hat{\Psi}(\boldsymbol{r}) \hat{\Phi}(\boldsymbol{r}):, \\
& \hat{H}_{\mathrm{CG}}=m \int d^{3} \boldsymbol{r} \hat{\Psi}^{\dagger}(\boldsymbol{r}) \hat{\Psi}(\boldsymbol{r}) \Phi[\Psi](t, \boldsymbol{r}),
\end{aligned}
$$

where :: refers to normal ordering, $m$ is the mass of the atoms, and we have made explicit that the classical potential $\Phi$ can be a functional of the quantum state $\Psi$ of the BEC, for which we have dropped a factor of $1 / 2$ [110]. Solving the quantized version of Poisson's equation, we have

$$
\hat{\Phi}(\boldsymbol{r})=-G m \int d^{3} \boldsymbol{r}^{\boldsymbol{r}^{\prime}} \frac{\hat{\Psi}^{\dagger}\left(\boldsymbol{r}^{\prime}\right) \hat{\Psi}\left(\boldsymbol{r}^{\prime}\right)}{\left|\boldsymbol{r}-\boldsymbol{r}^{\prime}\right|},
$$

where $G$ is the gravitational constant. In contrast, depending on the CG theory chosen, $\Phi$ is a certain quantum average of this expression (e.g., in the CG Schrödinger-Newton theory, which is the Newtonian limit of the semiclassical CG theory, $\Phi=\langle\hat{\Phi}\rangle$ [111-114]).

If we use $\hat{\Psi}(\boldsymbol{r})=\psi(\boldsymbol{r}) \hat{a}$, the above interaction Hamiltonians for the $\mathrm{BEC}$ reduce to

$$
\begin{aligned}
\hat{H}_{\mathrm{QG}} & =\frac{1}{2} \lambda_{\mathrm{QG}} \hat{a}^{\dagger} \hat{a}^{\dagger} \hat{a} \hat{a}, \\
\hat{H}_{\mathrm{CG}} & =\lambda_{\mathrm{CG}}[\Psi] \hat{a}^{\dagger} \hat{a},
\end{aligned}
$$


where $[115,116]$

$$
\begin{aligned}
\lambda_{\mathrm{QG}} & :=-G m^{2} \int d^{3} \boldsymbol{r} d^{3} \boldsymbol{r}^{\prime} \frac{\left|\psi\left(\boldsymbol{r}^{\prime}\right)\right|^{2}|\psi(\boldsymbol{r})|^{2}}{\left|\boldsymbol{r}-\boldsymbol{r}^{\prime}\right|}, \\
\lambda_{\mathrm{CG}}[\Psi](t) & :=G m \int d^{3} \boldsymbol{r}|\psi(\boldsymbol{r})|^{2} \Phi[\Psi](t, \boldsymbol{r}) .
\end{aligned}
$$

The QG interaction Hamiltonian (8) can also be derived as the nonrelativistic limit of linearized QG where we consider the four-point Feynman diagram with a single virtual graviton propagator, and then effectively integrate out gravitational degrees of freedom [108,117-121]. All QG theories must contain the above quantum Newtonian limit of gravity and so Eq. (8) is the Hamiltonian for general QG self-interactions of a BEC. Likewise, all CG theories must contain the above classical Newtonian limit of gravity for CG self-interactions of a BEC, and so Eqs. (8) and (9) are not dependent on a specific model of CG or QG. Similar Hamiltonians have been derived using cold atoms in a double-well potential [122], and the QG Hamiltonian (8) is analogous to the Kerr interaction, which induces non-Gaussianity in quantum optics [123].

From the Hamiltonians (8) and (9), we can see that as long as all other quantum interactions can be ignored (see Sec. III C), only the QG Hamiltonian (8) can induce nonGaussianity in the quantum state of the BEC field, with the CG Hamiltonian (9) preserving Gaussianity since it is quadratic in quantum operators. Therefore, any sign of non-Gaussianity being created in the BEC would be evidence of QG. In contrast, entanglement cannot be used as a witness here since this is just a single-mode system $[124,125]$.

\section{B. Measurement scheme}

As shown above, measuring creation of non-Gaussianity in the BEC would provide evidence of QG. To detect non-Gaussianity, we consider measurements of high-order cumulants [90]. For a Gaussian distribution, all cumulants higher than second order vanish and, therefore, a nonzero value of such cumulants is a signature of non-Gaussianity. Here we concentrate on the fourth-order cumulant $\kappa_{4}$, since $\kappa_{3}$ is also zero for a symmetric non-Gaussian distribution. Defining a generalized quadrature as $\hat{q}(\varphi)=\hat{a} e^{-i \varphi}+$ $\hat{a}^{\dagger} e^{i \varphi}$, we have

$$
\kappa_{4}:=\left\langle\hat{q}^{4}\right\rangle-4\langle\hat{q}\rangle\left\langle\hat{q}^{3}\right\rangle-3\left\langle\hat{q}^{2}\right\rangle^{2}+12\left\langle\hat{q}^{2}\right\rangle\langle\hat{q}\rangle^{2}-6\langle\hat{q}\rangle^{4} .
$$

In an experiment, only a finite sample can be used to estimate $\kappa_{4}$ and we desire unbiased estimators, which are the $k$ statistics: $\left\langle k_{n}\right\rangle=\kappa_{n}$ [126]. The noise in the estimation of $\kappa_{4}$ is then the standard deviation of $k_{4}$ [127], such that the signal-to-noise ratio (SNR) for the measurement is given by

$$
\left|\kappa_{4}\right| / \sqrt{\operatorname{var} k_{4}}
$$

where for a large number of independent measurements $\mathcal{M}, \operatorname{var} k_{4} \propto 1 / \mathcal{M}$.

To make the SNR as large as possible, we use quantum metrology, where highly quantum states can improve the estimation of parameters that are not associated with observables [128]. This is also effectively used in the BMV proposal, where the initial quantum states are N00N-like states $(|N 0\rangle+|0 N\rangle) / \sqrt{2}[122,129-131]$. However, rather than using a N00N state, here we consider a squeezed state, which is a Gaussian state that often provides similar performance in quantum metrology to N00N states but which is usually far less demanding to create [132]. Assuming that QG acts [i.e., that the gravitational interaction has the Hamiltonian of QG, Eq. (8)] and taking the limit that $\chi:=\left|\lambda_{\mathrm{QG}}\right| / \hbar$ is small and that the number of atoms $N$ of the BEC is large, we find that the SNR can be of order $\chi t N^{2} \sqrt{\mathcal{M}}$, where $t$ is the interaction time [133]. Assuming a weakly interacting BEC of mass $M$ in a spherical harmonic trap with frequency $\omega_{0}$, the $\mathrm{BEC}$ wave function is [61]

$$
\psi(\boldsymbol{r})=\frac{1}{\pi^{3 / 4} R^{3 / 2}} e^{-r^{2} / 2 R^{2}},
$$

where $R:=\sqrt{\hbar / m \omega_{0}}$ is the effective radius of the spherical BEC and $r:=|r|$. With use of Eq. (10), this results in [134]

$$
\chi t N^{2} \equiv \sqrt{\frac{2}{\pi}} \frac{G M^{2} t}{\hbar R},
$$

which is $t / \hbar$ times the gravitational self-energy of the BEC. With the replacement of $R$ with $d$, and ignoring the numerical factor, this expression is the same as the relative phase generated in the BMV proposal between the two microspheres that are separated by the smallest possible distance $d$ that, when all other distances are ignored, leads to an entangled state [27-30]. It is demonstrated in the BMV proposal that a value on the order of 1 for this phase is achieved when $d=200 \mu \mathrm{m}, t \approx 2 \mathrm{~s}$, and $M=10^{-14} \mathrm{~kg}$ [27]. However, since the SNR here scales with $\sqrt{\mathcal{M}}$, we can lower the total mass required by increasing the number of measurements. For example, to achieve a SNR of 5 for a ${ }^{133} \mathrm{Cs}$ BEC, we could use $R \approx 200 \mu \mathrm{m}, t \approx 2 \mathrm{~s}$, and $M=10^{-15} \mathrm{~kg}$ with around 40000 measurements. Such a mass corresponds to around $4 \times 10^{9}$ atoms, which is only a little larger than what has been achieved so far: in 1998 a ${ }^{1} \mathrm{H}$ BEC was created with more than $10^{9}$ atoms [135], and in 2006 a ${ }^{23} \mathrm{Na} \mathrm{BEC}$ had more than $10^{8}$ atoms [136]. However, the number of atoms required can be reduced by further increasing $\mathcal{M}$. 
An experimental implementation of this scheme would be to use a spin-1 BEC where the $m_{F}= \pm 1$ states are prepared in large coherent states and then a magnetic field is used to drive spin-mixing collisions to generate a quadrature squeezed state in the $m_{F}=0$ condensate. In a spin-1 $\mathrm{BEC}$, the interaction Hamiltonian is [137-139]

$$
\begin{gathered}
\hat{H}=\hbar \kappa\left[\hat{a}_{0}^{2} \hat{a}_{+}^{\dagger} \hat{a}_{-}^{\dagger}+\left(\hat{a}_{0}^{\dagger}\right)^{2} \hat{a}_{+} \hat{a}_{-}\right] \\
+\hbar \kappa\left(\hat{a}_{0}^{\dagger} \hat{a}_{0}-\frac{1}{2}\right)\left(\hat{a}_{+}^{\dagger} \hat{a}_{+}+\hat{a}_{-}^{\dagger} \hat{a}_{-}\right) \\
+\hbar q\left(\hat{a}_{+}^{\dagger} \hat{a}_{+}+\hat{a}_{-}^{\dagger} \hat{a}_{-}\right),
\end{gathered}
$$

where $\hat{a}_{0}$ is the annihilation operator of the $m_{F}=0$ mode and $\hat{a}_{ \pm}$are the annihilation operators of the $m_{F}= \pm 1$ modes. If $q$ is dynamically tuned with a magnetic field, the quadratic Zeeman shift (third term) cancels collisional shifts due to s-wave scattering of the three modes (second term) $[139,140]$. Then, if we further take the $m_{F}= \pm 1$ modes to be in large coherent states $\left(N_{ \pm} \gg 1\right)$ so that $\hat{a}_{ \pm} \approx \sqrt{N_{ \pm}}$, Eq. (16) acts as effectively

$$
\hat{H}=\hbar N \kappa\left[\hat{a}_{0}^{2}+\left(\hat{a}_{0}^{\dagger}\right)^{2}\right],
$$

where $N:=\sqrt{N_{+} N_{-}}$, which results in a single-mode quadrature squeezed state for the $m_{F}=0$ mode [141]. Spin-squeezing experiments have already been performed in cold atoms and BECs [59], where normally it is the $m_{F}=0$ mode that is taken to be the large coherent mode and then a two-mode squeezed state is created for the $m_{F}= \pm 1$ modes.

After the system has evolved for a time $t$, the nonGaussianity of the BEC field would then be measured. To achieve this, a homodyne or heterodyne scheme could be used [142-145], where moments up to fourth order are sought in the intensity difference, providing a direct map for obtaining $\kappa_{4}$. Observing a nonzero value for $\kappa_{3}$, which requires only the third-order moment in homodyne detection, would be sufficient for detecting non-Gaussianity, and the third-order correlation function of atoms due to electromagnetic self-interactions has already been measured in experiments [146]. However, $\kappa_{3}$ is predicted to be zero if the initial state of the BEC is a squeezed vacuum state, in which case $\kappa_{4}$ needs to be analyzed. For $\kappa_{4}$, the techniques used in Ref. [146] could be extended to measure the fourth-order correlations to obtain $\kappa_{4}$ through homodyne detection [123]. This would require single-atom counting in a quantum gas with high efficiency on small length scales, and recent advances have opened up very promising approaches to single-atom counting (see Appendix B 2 for more details). Rather than performing a homodyne or heterodyne measurement, another option would be to determine the Wigner function of the BEC, either using full state tomography with projective measurements (see, e.g., Refs. [142,147-150] for such measurements on cold atoms and BECs) or through "direct" measurement with weak measurements of the position quadrature and projective measurements of the momentum quadrature [151-154] (this has so far been achieved with photons [151,155-158] but could be extended to atoms [151]).

\section{Distinguishing quantum gravity from the electromagnetic interaction}

So far we have discussed how the desired input state can be generated and how non-Gaussianity could be measured. Additionally, it is imperative that we ensure that all noise can be distinguished from the signal. An advantage in considering a non-Gaussian signal is that we can immediately ignore all processes generating Gaussian noise since these will not affect the non-Gaussian measurement. The largest contributing non-Gaussian noise would be expected to come from the electromagnetic interactions between the atoms of the BEC. A BEC is very dilute and the atoms are neutral overall, but there are still, in general, weak electromagnetic interactions between the atoms due to van der Waals interactions and MDDIs. At the low temperatures at which BECs operate, the Hamiltonian for a BEC with electromagnetic interactions is [159-163]

$$
\begin{aligned}
\hat{H}= & \int d^{3} \boldsymbol{r}\left(-\frac{\hbar^{2}}{2 m} \hat{\Psi}^{\dagger}(\boldsymbol{r}) \nabla^{2} \hat{\Psi}(\boldsymbol{r})+V_{T}(\boldsymbol{r}) \hat{\Psi}^{\dagger}(\boldsymbol{r}) \hat{\Psi}(\boldsymbol{r})\right. \\
& +\frac{1}{2} \int d^{3} \boldsymbol{r}^{\prime}\left\{\hat { \Psi } ^ { \dagger } ( \boldsymbol { r } ) \hat { \Psi } ^ { \dagger } ( \boldsymbol { r } ^ { \prime } ) \hat { \Psi } ( \boldsymbol { r } ) \hat { \Psi } ( \boldsymbol { r } ^ { \prime } ) \left[g_{s} \delta^{(3)}\left(\boldsymbol{r}-\boldsymbol{r}^{\prime}\right)\right.\right. \\
& \left.\left.\left.+g_{d} \frac{1-3 \cos ^{2} \vartheta}{\left|\boldsymbol{r}-\boldsymbol{r}^{\prime}\right|^{3}}\right]\right\}\right)
\end{aligned}
$$

where the first term is the kinetic part, $V_{T}(\boldsymbol{r})=m \omega_{0}^{2} r^{2} / 2$ is the spherical trapping potential, $g_{s}:=4 \pi \hbar^{2} a_{s} / m$ is the s-wave scattering coupling constant, $g_{d}:=\mu_{0} \mu^{2} / 4 \pi$ parametrizes the strength of the MDDIs, and $\vartheta$ is the polar angle of $\boldsymbol{r}-\boldsymbol{r}^{\prime}$, with $a_{s}$ the s-wave scattering length, $\mu$ the magnetic moment of the atom, and $\mu_{0}$ the permeability of free space. With use of $\hat{\Psi}(\boldsymbol{r})=\psi(\boldsymbol{r}) \hat{a}$ and Eq. (14), the above Hamiltonian reduces to

$$
\hat{H}=\hbar \omega \hat{a}^{\dagger} \hat{a}+\frac{1}{2} \lambda_{s} \hat{a}^{\dagger} \hat{a}^{\dagger} \hat{a} \hat{a},
$$

where

$$
\begin{aligned}
\hbar \omega & :=\hbar \omega_{0}+\frac{3}{4} m \omega_{0}^{2} R^{2}, \\
\lambda_{s} & :=\frac{g}{2 \sqrt{2} \pi^{3 / 2} R^{3}} \equiv \sqrt{\frac{2}{\pi}} \frac{a_{s} \hbar^{2}}{m R^{3}} .
\end{aligned}
$$

The MDDIs have canceled out due to the spherical symmetry of the BEC, leaving behind only the s-wave interactions 
$[160,162]$. This interaction term has the same form as the quantum gravitational interaction (8), and so we need to be able to distinguish between the electromagnetic and gravitational interactions in order to attribute non-Gaussianity to only gravitational interactions. One way to achieve this is to use magnetic or optical Feshbach resonances, which are extraordinary processes particular to cold atom and BEC experiments. Here an external magnetic or optical field is used to resonantly couple a molecular bound state to a colliding atom pair, enabling the strength of the electromagnetic interactions to be controlled $[60,61]$.

Usually Feshbach resonances are used to increase the electromagnetic interaction strength between atoms in BECs. However, they also allow the electromagnetic interaction to be, in principle, switched off, (i.e., $\lambda_{s}=0$ ) without affecting the strength of the gravitational interaction. This is achieved by applying a magnetic field of strength $B$ to the BEC, which results in the s-wave scattering length becoming a function of $B[60,61]$ :

$$
a_{s}(B)=a_{s}^{\mathrm{BG}}\left(1-\frac{\Delta}{B-B_{0}}\right),
$$

where $a_{s}^{\mathrm{BG}}$ is the background scattering length, $B_{0}$ denotes the resonance position, and $\Delta$ is the resonance width. The s-wave interactions can then be turned off by setting $B=B_{0}+\Delta[164]$. For ${ }^{133} \mathrm{Cs}$, this would be achieved when $B=17 \mathrm{G}[165,166]$, leaving behind only the QG interactions (8), which are unaffected by the applied magnetic field. With the electromagnetic interactions in the BEC turned off, non-Gaussianity can, in principle, be attributed solely to QG interactions in the BEC [167].

\section{DISCUSSION}

\section{A. Role of Planck mass in the proposed experiment}

We have argued that, as long as we are working in a situation where all other nongravitational quantum interactions can be excluded, the production or change in non-Gaussianity in the state of the quantum field of matter would be sufficient evidence of QG, and we have demonstrated how this could be used in a test that is based on just a single-well BEC. The size of the effect in the BEC experiment appears to be similar to that observed in the BMV proposal; see Eq. (15) and Refs. [27-30]. This illustrates how the experiment is related to the Planck mass since, using Eq. (15), we can write the SNR for one measurement in this case as $[22,168]$

$$
\frac{M}{M_{P}} \frac{\delta \tau}{t_{P}}
$$

where $M_{P}$ is the Planck mass, $t_{P}$ is the Planck time, and $\delta \tau:=\sqrt{2 / \pi} G M t / R c^{2}$. This expression can also be derived by dividing the BEC into two halves, considering the gravitational interaction of one with the other and the time dilation $\delta \tau$ induced in GR in the center of each half. If we fix this SNR of one measurement, then Eq. (23) illustrates that as $M$ gets closer to $M_{P}$, it seems that we can probe more minute gravitational field intensities and thus further access its possible quantum properties.

\section{B. Direct classical gravity interactions can create entanglement between two quantum field systems but not non-Gaussianity}

A classical interaction can create entanglement if this involves the respective quantum systems directly interacting with each other [27-30]. For example, consider two BECs that are in the two spatial arms of a doublewell potential. In the two-mode approximation, we can write the full quantum field of the atoms as $\hat{\Psi}(\boldsymbol{r})=$ $\psi_{L}(\boldsymbol{r}) \hat{a}_{L}+\psi_{R}(\boldsymbol{r}) \hat{a}_{R}$, where $\hat{a}_{L}$ and $\hat{a}_{R}$ destroy an atom in the left well and an atom in the right well, respectively, and $\psi_{L}$ and $\psi_{R}$ are the corresponding mode wave functions $[61,169]$. In the case of CG, and taking the Newtonian approximation for simplicity, there will, in principle, be terms of the form $\lambda_{L R} \hat{a}_{L}^{\dagger} \hat{a}_{R}+$ h.c. in the Hamiltonian, where $\lambda_{L R}:=m \int d^{3} \boldsymbol{r} \psi_{L}^{*}(\boldsymbol{r}) \psi_{R}(\boldsymbol{r}) \Phi[\Psi](\boldsymbol{r}, t)$. These are beam-splitting terms such that if $\lambda_{L R}$ is nonzero due to, for example, the mode wave functions overlapping, and either BEC is in a nonclassical state, then the terms will induce entanglement between the BECs. There is an electromagnetic analogue of this effect where a double-well trapping potential, which is approximated to be classical, causes or contributes to entanglement between the two wells. This entangling process is often referred to as "quantum tunneling" in cold atom experiments [61]. However, since the entangling-inducing terms are quadratic, they will not induce non-Gaussianity, illustrating that although a direct classical interaction with matter can create entanglement, it cannot create non-Gaussianity in the quantum field of matter.

Note that here we are working with "mode" entanglement (i.e., entanglement between modes of a quantum field). If instead we attempted to use a first-quantization picture and describe the full system using a many-body wave function, then it is possible to argue that the initial state of the full system is already entangled and that Newtonian CG is not creating entanglement in this picture [56-58]. This is because there is so-called particle entanglement before and after the effective CG beam splitter $[132,170]$. For example, the initial state could be $|\alpha\rangle_{L}|\xi\rangle_{R}$, with $|\alpha\rangle$ a coherent state and $|\xi\rangle$ a squeezed state, which, in a quasi first-quantization picture, is particle entangled but not mode entangled [132]. This occurs because in the firstquantization picture a beam splitter does not couple the left and right wells. However, in the relativistic CG limit we would also have, in principle, two-mode squeezing operations such as $\hat{a}_{L} \hat{a}_{R}+\hat{a}_{L}^{\dagger} \hat{a}_{R}^{\dagger}$, which can result in a twomode squeezed state, which is particle entangled [132]. 
Therefore, in full generality and in either picture, CG can, in principle, create entanglement. In contrast, in the first-quantization picture, it is possible for Newtonian CG to create non-Gaussian "particle" Wigner functions. For example, the many-body wave function of our single-well BEC experiment could start off Gaussian but become nonGaussian under CG (see Appendix A 2 a for more details). However, in the more fundamental second-quantization picture, the state of matter (i.e., the state of the quantum field of matter) always remains Gaussian under CG, as shown in Sec. II.

\section{Nonquantum interactions and continuous-time measurements}

Above we defined a classical interaction as an interaction with an entity $\mathcal{G}$ that takes on real and well-defined values, such as the gravitational field of GR. We now consider whether non-Gaussianity can also be used to distinguish other, more general, nonquantum interactions from their quantized counterparts. First we consider that $\mathcal{G}$ takes on complex values. This allows the possibility that, most generally, the interaction can give rise to a Hamiltonian of the form of Eq. (2) but where now the coupling constants $\lambda_{k}$ and $\boldsymbol{\mu}_{k}$ are complex valued. Although this, in general, leads to a non-Hermitian Hamiltonian, a matter state with a Gaussian Wigner function will continue to have a Wigner function of Gaussian form [171-173], and so non-Gaussianity can also distinguish this interaction from a quantum interaction [174].

Another possibility is that $\mathcal{G}$ could be a nonquantum but stochastic quantity. For example, a relativistic theory of gravity coupled to matter has been proposed where the nonquantum gravitational field is stochastic [175]. It is found that gravity and matter interact through a Gaussian completely positive channel, and so non-Gaussianity should also rule out this nonquantum theory of gravity [176]. More generally, interacting a stochastic entity $\mathcal{G}$ with a quantum field will still result in a Gaussian state of the quantum field remaining Gaussian if we now broaden our definition of a Gaussian state to include states that are a statistical mixture of pure states with Gaussian Wigner functions (the so-called Gaussian convex hull $[92,177]$ ). This is because a Gaussian state evolves to a state in the Gaussian convex hull if there is a combination of Gaussian operations and statistical randomization (i.e. stochasticity) [93], see Appendix F for more details.

The preservation of this broader definition of Gaussianity also applies if the entity $\mathcal{G}$ is both stochastic and complex valued. However, in this case the norm will not, in general, be preserved, and so we have a mixture of unnormalized states with Wigner functions of Gaussian form [171-173]. To ensure that the theory is norm preserving, the physical state vector can be redefined as
$|\Psi\rangle / \| \Psi\rangle$, which then allows for a convex mixture of properly normalized Gaussian states. However, this, in general, results in a theory that is nonlinear in the density matrix, leading to superluminal signaling [179]. Such an issue is also found in objective-collapse theories, and to rectify it, a new higher-order process is applied to the evolution of the quantum system $[180,181]$, which would here be associated with a quantum (self) interaction of matter (i.e., a new force): see Appendix F for more details, and in particular, Appendix F 2. For example, if we represented matter by a real scalar field $\hat{\phi}$, the Hamiltonian of this new process would involve terms that are quartic in $\hat{\phi}$. This new quantum process can, in general, induce non-Gaussianity. However, in the conventional case that the noise term of the objective-collapse theory has a Gaussian profile and is anti-Hermitian (equivalent here to only the imaginary component of $\mathcal{G}$ being stochastic), Gaussianity in the matter field is still preserved. This is also analogous to a continuous-time measurement being performed on matter by the stochastic entity $\mathcal{G}$, which could be a stochastic gravitational field, and the new quantum self-interaction [182-185].

\section{Alternative theories of gravity}

Einstein's GR can be formulated as an action theory with the action principle being used to derive the field equations. The action $S$ of GR can be decomposed into the Einstein-Hilbert action $S_{\mathrm{EH}}$, which contains only gravitational degrees of freedom, and the matter action $S_{M}$, which tells us how matter and gravity interact:

$$
S=S_{\mathrm{EH}}+S_{M},
$$

where

$$
S_{\mathrm{EH}}=\frac{c^{4}}{16 \pi G} \int d^{4} x \sqrt{g} R,
$$

with $R$ the Ricci scalar, and for a real scalar matter field $\phi$,

$$
S_{M}=\frac{1}{2} \int \sqrt{g}\left[g^{\mu \nu} \partial_{\mu} \phi \partial_{\nu} \phi-\left(m^{2}+\varepsilon R\right) \phi^{2}\right],
$$

with $\varepsilon$ a numerical factor. The matter actions for spin- $1 / 2$ and spin-1 fields are provided in Appendix A.

The argument that we have presented for nonGaussianity being an indicator of QG uses only the matter action $S_{M}$ and says nothing of the purely gravitational action $S_{\mathrm{EH}}$. It relies on the fact that $S_{M}$ must be, in the absence of all nongravitational quantum interactions, quadratic in the matter fields such that a classical theory of gravity will preserve Gaussianity. If $S_{M}$ had terms that coupled gravitational degrees of freedom with nonlinear or nonquadratic functions of the matter fields, then in flat spacetime such terms would still exist and this would 
result in a new nongravitational interaction, which we have excluded.

Many theories of gravity have been suggested as alternatives to Einstein's GR [186]. These tend to consider alternative forms for the gravitational action $S_{\mathrm{EH}}$. For example, in $f(R)$ theories of gravity [187], the $R$ in $S_{\mathrm{EH}}$ is replaced with some function of the Ricci scalar $f(R)$. With use of the argument above, as long as we can exclude all other relevant quantum interactions, non-Gaussianity can still be used as evidence of a quantum version rather than a classical version of these alternative theories of gravity.

\section{E. Non-Gaussianity in cosmology}

Non-Gaussianity is often considered in the context of cosmology. Here studies predominately concentrate on how the temperature fluctuations of the cosmic microwave background (CMB) could follow a non-Gaussian distribution. So far measurements are consistent with a Gaussian distribution, but with the ever-increasing precision of CMB measurements, it is possible that non-Gaussianity could be detected soon, providing important insights into structure formation in our universe.

Perhaps the most important mechanisms responsible for generating a non-Gaussian distribution of temperature fluctuations are inflationary mechanisms, which involve processes that occur at the surface of last scattering or before. These are often referred to as "primary effects" or "primordial effects," and can be further subcategorized into quantum mechanical effects at or before horizon exit and classical nonlinear effects after horizon exit (see, e.g., Ref. [188]). The most primitive physical mechanism for inflation assumes a single scalar field, the inflaton, that couples to gravity and acquires a nonzero vacuum expectation value, leading to exponential expansion of space. The Hamiltonian for this model is similar to the one we used to illustrate that non-Gaussianity in the quantum state of matter can be used as evidence of QG [see Eqs. (3) and (A5)]. An important difference, however, is that the inflaton, unlike normal matter (Standard Model leptons and quarks), has a nonquadratic potential $V(\hat{\phi})$ and thus self-interacts.

If the vacuum state is initially assumed, then at second order in quantum fluctuations of the inflaton and gravitational fields, a squeezed Gaussian state of curvature perturbations is created, which is the leading-order effect and can explain the Gaussian nature of the CMB that has been observed so far (see, e.g., Refs. [188,189]). At the next order, non-Gaussian effects occur due to the coupling between QG and the inflaton as well as self-interactions of the inflaton [190]. The former effect is analogous to the interaction we have considered between QG and matter that generates non-Gaussianity in the quantum state of matter. The latter effect means that, in principle, it would be possible for purely classical gravitational effects to enhance non-Gaussianity in this inflaton model since gravity will couple to the non-Gaussian-generating selfinteraction of the inflaton. This further illustrates that only if we are able to work in a situation where all other quantum mechanical interactions can be ignored can we use non-Gaussianity as evidence of a quantum theory of gravity. In this case, we are not ignoring the quantum self-interaction of the inflaton. Furthermore, unless a proper measure of non-Gaussianity is used, then even if we can ignore the inflaton self-interactions, CG effects can give the appearance of enhancing any already present non-Gaussianity in the inflaton. This is because the Hamiltonian (3) can lead to an increase or a decrease in higherorder cumulants, such as $\kappa_{4}$, if the scalar field is already in a non-Gaussian state. However, when a proper measure of non-Gaussianity is used, such as the SNR defined in Eq. (13) or that based on quantum relative entropy [86], these measures do not change, and so non-Gaussianity is not really increasing or deceasing.

As mentioned above, non-Gaussianity in quantum curvature perturbations can also occur after horizon exit. In this case, a nonlinear classical evolution can result in a nonlinear relationship between the quantum curvature perturbations and the inflaton field, which results in nonGaussian statistics for the curvature perturbations even if the inflaton is in a Gaussian state (see, e.g., Ref. [188]). Here the non-Gaussianity is in the quantum curvature perturbations not in the inflaton field, which, if we ignore its self-interactions, is analogous to the matter field $\phi$ we use in Sec. II to illustrate our argument that CG cannot create non-Gaussianity in the quantum field state of matter.

The reason that non-Gaussianity in the quantum state of curvature perturbations is related to non-Gaussianity in the temperature fluctuations of the CMB is due to the SachsWolfe effect [191]. This is a classically treated effect where the curvature fluctuations result in redshifts of the radiation such that there is a direct map between correlation functions of the curvature perturbations and correlation functions of temperature fluctuations in the CMB. Any non-Gaussianity in the CMB due to a single-field model of inflation is expected to be very small and outside the realms of near-future observations of the CMB. Instead, more complex models are needed for observable levels of non-Gaussianity, such as multifield inflation [192]. Detection of non-Gaussianity would, therefore, potentially provide crucial knowledge of the mechanisms responsible for structure formation.

However, there are many other mechanisms responsible for creating a non-Gaussian distribution of temperature fluctuations in the CMB besides the inflationary ones. These include so-called secondary effects, which involve processes that occur between the last scattering surface and the observer (see, e.g., Refs. [193,194]). Secondary effects can be broadly divided into scattering of the CMB radiation with hot gas and classical effects mediated by 
gravity subsequent to the last scattering surface, such as the time-integrated Sachs-Wolfe effect [191] and gravitational lensing. Other effects that can be responsible for nonGaussian temperature fluctuations include nonlinear effects at recombination (see, e.g., Refs. [193,194]). Although detection of these nonprimordial effects would provide important information for distinguishing structure formation scenarios, they are often regarded as noise associated with the primary inflationary effects.

It is thought that measurements of the CMB could provide evidence for a quantum theory of gravity. For example, QG predicts that there will be tensor modes due to quantum fluctuations of the gravitational field during inflation. Dimensional arguments involving Planck's constant can then potentiality be used for evidence of QG if such modes are observed [195]. Other possibilities have also been suggested, such as using measurements of the scalar modes of the temperature fluctuations to try to access quantum measures of the primordial curvature perturbations, such as violation of Bell inequalities [196] or quantum discord [197]. The issue here, however, is that we have access only to the field modes, not their momentum conjugate, which is part of the "decaying mode," and we can measure only one instance of the CMB at a time. There then does not seem to be enough information to rule out classical curvature perturbations [196-198], and instead rather convoluted inflationary models need to be assumed for evidence of QG [198].

If we were to brute forcefully apply our non-Gaussianity argument for evidence of QG to cosmology, then this would require measuring the quantum state of the $\mathrm{CMB}$ radiation and somehow being able to distinguish gravitational interactions with the CMB from nongravitational ones, such as secondary effects due to scattering with electrons. In this case, assuming that the initial quantum state of the CMB is Gaussian, any sign of non-Gaussianity in its quantum state due to gravitational interactions would be evidence of a quantum theory of gravity. Unfortunately, measuring the temperature fluctuations of the CMB radiation does not, in general, provide information on the quantum state of the radiation. For example, if we were to consider an ideal gas of radiation in a container in a static curved spacetime, then at thermal equilibrium the radiation has Bose-Einstein statistics [199,200] and the quantum state of the radiation is a Gaussian thermal state (see, e.g., Ref. [54]). However, because of the form of the spacetime metric and the Ehrenfest-Tolman effect $[201,202]$, the radiation can have temperature fluctuations in space that obey a non-Gaussian distribution. Therefore, non-Gaussian fluctuations of the temperature of the $\mathrm{CMB}$ do not necessarily mean that the quantum state of the CMB itself is non-Gaussian. Any fundamentally classical gravitational effects that generate non-Gaussianity in the temperature fluctuations of the CMB will not change the non-Gaussianity in the quantum state of the radiation.
This applies in the perturbative regime as well as the nonperturbative regime of the gravity since our argument is just based on the fact that the CG Hamiltonian is quadratic in the quantized matter fields, which is the case in both the perturbative regime and the nonperturbative regime of gravity (this also means that the CG Hamiltonian can be solved in the absence of standard quantum perturbation theory - see Appendix E). Instead, quantum gravitational mechanisms would need to be considered, such as a quantum version of the Sachs-Wolfe effect, for changing the non-Gaussianity of the quantum state of the CMB radiation due to gravity.

Measurements of temperature fluctuations of the CMB are thought to provide information on the quantum state of the primordial gravitational field through the classical Sachs-Wolfe effect. In contrast, if we were able to measure the non-Gaussianity of the quantum state of the CMB radiation and robustly claim that this is due to gravitational interactions, then this would provide evidence of QG through an indirect means of analyzing the quantum state of radiation, with no knowledge of the state of the primordial gravitational field being required.

\section{F. Applicability}

We have argued that, when representing matter with a quantum field, non-Gaussianity in its quantum state can be used as an indirect signature of a quantum rather than a classical theory of gravity. In certain theories of QG, such as loop QG, group field theory [203,204], and asymptotically safe QG $[205,206]$, matter is fundamentally described by quantum fields. However, in other QG theories, such as string theory, this representation of matter is a limiting lowenergy description of that used in the fundamental theory, and the low-energy description is referred to as "effective field theory" [207,208]. In this case, if we wanted to use a notion of non-Gaussianity that is applicable to the representation used in the full theory, our concept of non-Gaussianity in matter would have to be updated, or it may only be applicable to the low-energy effective field theory description. Our argument stems from the fact that the Hamiltonian or action of gravity in the quantum field regime of matter has matter-gravity terms that are quadratic only in the quantum matter operators. There is a connection here with string theory (see the Polyakov action in curved space [8]), suggesting that our notion of nonGaussianity in matter could also be generalized to strings. However, in foreseeable tabletop tests of QG, it is very unlikely that anything beyond the (potentially effective) quantum field theory description QG will be probed.

\section{SUMMARY}

We have shown that if we want to create nonGaussianity in the quantum field state of matter with purely gravitational interactions, then this would not be possible 
with a classical theory of gravity but can be achieved with a quantum theory of gravity. On the theoretical side, this opens up a connection between QG and QIS. Perhaps the most important property of this connection is that, in contrast to other quantum information resources such as entanglement, although QG can create non-Gaussianity, CG can never create non-Gaussianity in the quantum field of matter (as long as, as with entanglement, all other quantum interactions can be ignored). For example, while a classically expanding spacetime metric can create entanglement in the quantum field of matter [36-43], it cannot create non-Gaussianity. This also suggests that whereas entanglement is not invariant to changes in classical reference frames [209], non-Gaussianity and nonclassicality are.

Non-Gaussianity is a very important resource in QIS. For instance, it is necessary for universal quantum computation $[48,49]$. However, it is not sufficient for universal speedup over classical computation. For this, we need negative Wigner function states, and in the case of mixed states, it is possible for a non-Gaussian state to have a positive Wigner function. Given that it is negative Wigner function states that are generically associated with nonclassicality, it is interesting from a fundamental point of view that it appears to be non-Gaussianity that is a universal indicator of QG rather than negative Wigner function states. Perhaps non-Gaussianity in matter, especially its broader definition of states outside the Gaussian convex hull, is connected with a more fundamental property of quantum gravitational degrees of freedom, such as non-commutating variables or quantum contextuality.

Approaching QG from a quantum information perspective has attracted much theoretical interest recently, especially in regard to uncovering the role that quantum correlations, such as entanglement, may play in the fundamentals of QG. Conventionally, QG has been considered in the context of discrete-variable quantum information, such as qubits, whereas here we have concentrated on describing QG using continuous-variable quantum information, and resources particular to CVQIS. Just as CVQIS has been extremely effective in connecting quantum information and quantum field theory, we find that it is also very powerful in connecting quantum information and QG. Our findings, however, could also potentially be extendable to describing QG with discrete-variable quantum information since the Wigner function can also be defined for discrete systems [210-213].

As well as providing a theoretical link between QG and QIS, we have shown how non-Gaussianity can be used to implement new types of experimental tests of QG. In particular, non-Gaussianity allows tests based on just a single quantum system rather than a multipartite quantum system, and it also provides a particularly reliable signature of QG since it cannot be created by indirect or direct CG interactions (as long as all other interactions can be ignored). This is in contrast to previous tests based on entanglement where a multipartite quantum system is necessary and where entanglement is an indicator of QG only in certain contexts, allowing, in principle, for certain loopholes in which CG could be creating the expected QG signal, such as nonlocal effects or direct CG interactions that occur due to overlapping mode wave functions (see Sec. IV B).

We have proposed a tabletop test of QG that uses our non-Gaussianity witness and current technology. This proposal uses a $4 \times 10^{9}$ atom BEC in just a single-well potential, with $10^{9}$-atom BECs having already been created in single wells [135]. The most promising proposal so far for a tabletop test of QG is considered to be the BMV proposal, which, in contrast to our quantum gas experiment, uses an optomechanical setup and entanglement as a witness of QG. In our proposal and the BMV proposal, the QG signal scales quadratically with the mass of the system and linearly with the interaction time. The signal in both proposals is greatest when the initial state is a highly nonclassical state: here we use a squeezed state, whereas the BMV effectively uses a N00N state, which is considered to be the most challenging quantum state to create. So far, neither N00N states nor squeezed states in the quantum regime have been created in nanoparticle or microparticle experiments, whereas squeezed states in the quantum regime have been created in BECs [59], further facilitating the implementation of our proposal. However, macroscopic quantum squeezed states have yet to be generated. We have investigated how these could be achieved in the near term, but, just as with other proposed tabletop tests of QG that use QIS [23,26-31,33], creating such macroscopic states is an experimental challenge in realizing the experiment. Another option would be to use a classical coherent initial state but increase the mass of the system and the number of repetitions of the experiment by 1 or 2 orders of magnitude, which will be considered in future work.

As with other recently proposed QIS tabletop tests of QG [23,25-33], we need to ensure that the electromagnetic interactions can be suppressed or distinguished from the gravitational interactions. In the BMV proposal, for example, this means increasing the separation between the microspheres, which also lowers the gravitational signal, as well as ensuring that there is no excess charge on either microsphere. An advantage in using quantum gases is that the electromagnetic interactions are manipulable using external magnetic or optical fields [61], which allows the noise from electromagnetic interactions to be suppressed without affecting the strength of the gravitational interactions. We have considered a ${ }^{133} \mathrm{Cs}$ BEC since this has the broadest and strongest Feshbach resonance, allowing, in principle, sufficiently low levels of electromagnetic noise in the experiment.

BECs and cold atoms have been found to be very effective in tests of classical gravity, and experiments using 
these are now becoming the state of the art for many types of classical gravity measurements [214]. Their effectiveness can be attributed to the extraordinary degree of control that BECs and cold atoms provide in exploring the fundamental behavior of quantum matter in various settings. In particular, Feshbach resonances enable control of the electromagnetic interactions between atoms, providing a key tool that has led to several scientific breakthroughs $[60,61]$. Given their great success in classical gravity measurements and the degree of control offered by these systems, it is perhaps not surprising that we find that BECs could also be very useful for measuring quantum gravitational effects.

\section{ACKNOWLEDGMENTS}

We thank Chiara Marletto, Andrea Di Biaggio, and participants of the University of Hong Kong Quantum Information Structure of Spacetime (QISS) 2020 workshop and the Physical Institute for Theoretical Hierarchy for stimulating and insightful discussions. R.H. acknowledges ERC StG GQCOP (Grant Agreement No. 637352), as well as Gerardo Adesso and Giulio Chiribella for their great support. R.H. also acknowledges Jan Robbe for generously allowing the use of his artwork "Space and Particles 8" for a creative visualization of the proposed BEC experiment. V.V., M.C., and C.R. acknowledge the support of Grant No. 61466 from the John Templeton Foundation as part of the QISS project. The opinions expressed in this publication are those of the authors and do not necessarily reflect the views of the John Templeton Foundation. D.N. acknowledges the support of the Agence Nationale pour la Recherche (EOSBECMR, Grant No. ANR-18-CE910003-01).

\section{APPENDIX A: NON-GAUSSIANITY IN QUANTUM GRAVITY}

The way in which matter and gravity interact in GR is described by the matter action $S$, which can be derived from the specific Lagrangian density $\mathcal{L}(x)$ for the matter field:

$$
S=\int d^{4} x \mathcal{L}(x)
$$

For example, if all other interactions (which automatically include any self-interactions) are ignored, then in the metric or tetrad formulations of GR, the respective Lagrangian densities for a real scalar $\phi$, spin-1/2 $\psi$, and spin-1 field $A_{\mu}$ are $[39,102]$

$$
\begin{aligned}
\mathcal{L}_{\phi} & =\frac{1}{2} \sqrt{g}\left[g^{\mu \nu} \partial_{\mu} \phi \partial_{\nu} \phi-\left(m^{2}+\varepsilon R\right) \phi^{2}\right] \\
& \equiv \frac{1}{2} e\left[\eta^{\alpha \beta} e_{\alpha}^{\mu} \partial_{\mu} \phi e_{\beta}^{\nu} \partial_{\nu} \phi-\left(m^{2}+\varepsilon R\right) \phi^{2}\right],
\end{aligned}
$$

$$
\begin{aligned}
& \mathcal{L}_{\psi}=\sqrt{g}\left\{\frac{1}{2} i\left[\bar{\psi} \gamma^{\mu} \nabla_{\mu} \psi-\left(\nabla_{\mu} \bar{\psi}\right) \gamma^{\mu} \psi\right]-m \bar{\psi} \psi\right\} \\
& \mathcal{L}_{A}=-\frac{1}{4} \sqrt{g} g^{\mu v} g^{\nu \sigma} F_{\mu \rho} F_{v \sigma},
\end{aligned}
$$

where $e_{\alpha}^{\mu}(x)$ are tetrads, the "matrix square root" of the metric tensor: $g^{\mu \nu}(x)=: e_{\alpha}^{\mu}(x) e_{\beta}^{\nu}(x) \eta^{\alpha \beta}$, with $\mu$ labeling the general spacetime coordinate, $\alpha$ the local Lorentz spacetime, and $\eta^{\alpha \beta}$ the Lorentz metric. Furthermore, $F_{\mu \nu}:=\partial_{\nu} \mathcal{A}_{\mu}-\partial_{\mu} \mathcal{A}_{\nu}$ is the electromagnetic tensor, $\mathcal{A}_{\mu}$ is the electromagnetic four potential, $R$ is the Ricci scalar, $\nabla_{\mu}$ is the covariant derivative, $\gamma^{\mu}:=e_{\alpha}^{\mu} \gamma^{\alpha}$ are the curved space counterparts of the (Dirac) $\gamma$ matrices, which satisfy $\left\{\gamma^{\mu}, \gamma^{\nu}\right\}=2 g^{\mu \nu}, \varepsilon$ is a numerical factor that we set to zero for the rest of this appendix for simplicity, and the metric signature chosen is $(-,+,+,+)$. For a complex rather than a real scalar field, we replace terms with two copies of $\phi$ by one copy of $\phi^{*}$ and $\phi$; for example, $\partial_{\mu} \phi \partial_{\nu} \phi$ becomes $\partial_{\mu} \phi^{*} \partial_{\nu} \phi$.

We can also write corresponding Hamiltonian (constraint) densities for the above Lagrangian densities [14, 101,102]:

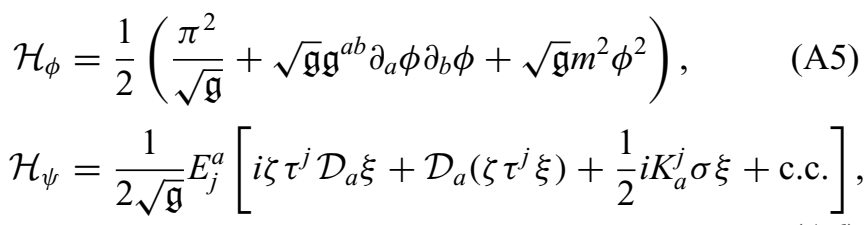

$\mathcal{H}_{A}=\frac{1}{2 \sqrt{\mathfrak{g}}} \mathfrak{g}_{a b}\left(\mathcal{E}^{a} \mathcal{E}^{b}+\mathcal{B}^{a} \mathcal{B}^{b}\right)$

Here spacetime has been split into spatial slices and a time axis $M=\mathbb{R} \times \sigma$. Taking $n^{\mu}$ to be the normal vector field of the time slices $\sigma$, we can write the tetrad as $e_{\alpha}^{\mu}=\mathfrak{e}_{\alpha}^{\mu}-n^{\mu} n_{\alpha}$, with $\eta^{\alpha \beta} n_{\alpha} n_{\beta}=-1$ an internal unit timelike vector (which we may choose to be $n_{\alpha}=-\delta_{\alpha, 0}$ ), so that $\mathfrak{e}_{\alpha}^{\mu}$ is a triad, where $\mathfrak{e}_{\alpha}^{\mu}=\left(0, \mathfrak{e}_{i}^{\mu}\right)$ and we further define $E_{i}^{a}=\left|\operatorname{det} \mathfrak{e}_{i}^{a}\right| \mathfrak{e}_{i}^{a}$ with $i, a=1,2,3$. The momentum conjugate to the densitized triad $E_{i}^{a}$ is the chiral spin connection $A_{a}^{i}:=\Gamma_{a}^{i}+K_{a}^{i}$, where $\Gamma_{a}^{i}=\Gamma_{a j k} \epsilon^{j k i}$ and $K_{a}^{i}=$ $K_{a b} \mathfrak{e}^{b i}$, with $\Gamma_{a j k}$ the spin connection and $K_{a b}$ the extrinsic curvature. In Eqs. (A5)-(A7), $\mathfrak{g}$ is then the determinant of the induced spatial metric $\mathfrak{g}_{a b}=\mathfrak{e}_{i}^{a} \mathfrak{e}_{j}^{b} \delta^{i j}$ on the spatial slices, $\pi:=\sqrt{\mathfrak{g}} n^{\mu} \partial_{\mu} \phi$ is the momentum conjugate to $\phi$, $\mathcal{E}^{a}:=\sqrt{\mathfrak{g} \mathfrak{g}^{a b}} n^{\mu} F_{\mu b}$ is the electric field, $\mathcal{B}^{a}:=\epsilon^{a b c} F_{b c}$ is the magnetic field, $\tau_{i}$ are the generators of the Lie algebra $\operatorname{su}(2)$ with the convention $\left[\tau_{i}, \tau_{j}\right]=\epsilon_{i j k} \tau_{k} ; \xi=\sqrt{\mathfrak{g}} \psi$, with $\psi$ a Grassman-valued fermion field, $\zeta$ is the momentum conjugate to $\xi$, and $\mathcal{D}_{a} \xi:=\left(\partial_{a}+\tau_{j} A_{a}^{j}\right) \xi$. For simplicity, we also assume that the scalar and fermionic fields are singlets under any internal group symmetry. 
Since we have ignored all other interactions, the above Lagrangian and Hamiltonian densities are all necessarily quadratic in matter fields as they then consist of only kinetic and mass terms. This quadratic scaling of course applies to any spin field not just those considered above [215-223]. Therefore, if we quantize the matter fields but leave the gravitational degrees of freedom classical, we have a theory that preserves Gaussianity. However, if gravity obeys a quantum theory, then there must be some quantum operator associated with it, and, therefore, we must have a theory that has interactions involving three or more quantum operators and that thus induces nonGaussianity. In the next two subsections we also illustrate this argument in the weak-field and nonrelativistic limits of gravity.

\section{Weak-field limit}

In the weak-field limit of gravity, we write $g_{\mu \nu}=\eta_{\mu \nu}+$ $h_{\mu \nu}$, where $h_{\mu \nu}$ is a perturbation around a spacetime background with metric $\eta_{\mu \nu}$. In this case, the GR matter-gravity interaction Hamiltonian is [224]

$$
H_{\mathrm{int}}=-\frac{1}{2} \int d^{3} \boldsymbol{r} T^{\mu v} h_{\mu \nu}
$$

where

$$
\square h_{\mu \nu}=\frac{16 \pi G}{c^{4}}\left(\frac{1}{2} \eta_{\mu \nu} \eta^{\sigma \rho} T_{\sigma \rho}-T_{\mu \nu}\right),
$$

with $\square$ the d'Alembert operator and $T^{\mu v}$ the stress-energy tensor for matter. The stress-energy tensor for a field of arbitrary spin in curved spacetime can be obtained by variation of the action with respect to the metric [39]:

$$
T_{\mu \nu}(x)=\frac{2}{\sqrt{-g(x)}} \frac{\delta S}{\delta g^{\mu \nu}(x)} \equiv \frac{e_{\alpha \mu}(x)}{e(x)} \frac{\delta S}{\delta e_{\alpha}^{\mu}(x)} .
$$

For example, when all other interactions are ignored, for a real scalar, spin-1/2, and spin-1 field, the curved space stress-energy tensors are (before taking a weak-field limit) [39]:

$$
\begin{gathered}
T_{\mu \nu}^{\phi}=(1-2 \varepsilon) \partial_{\mu} \phi \partial_{\nu} \phi+\left(2 \varepsilon-\frac{1}{2}\right) g_{\mu \nu} g^{\rho \sigma} \partial_{\rho} \phi \partial_{\sigma} \phi-2 \varepsilon\left(\nabla_{\mu} \partial_{\nu} \phi\right) \phi+\frac{1}{2} \varepsilon g_{\mu \nu} \phi \square \phi \\
-\varepsilon\left[R_{\mu \nu}-\frac{1}{2} R g_{\mu \nu}(1-3 \varepsilon)\right] \phi^{2}+\frac{1}{2}(1-3 \varepsilon) m^{2} g_{\mu \nu} \phi^{2}, \\
T_{\mu \nu}^{\psi}=\frac{1}{2} i\left[\bar{\psi} \gamma_{(\mu} \nabla_{\nu)} \psi-\left[\nabla_{(\mu} \bar{\psi}\right] \gamma_{\nu)} \psi\right], \\
T_{\mu \nu}^{A}=\frac{1}{4} g_{\mu \nu} F^{\rho \sigma} F_{\rho \sigma}-F_{\mu}^{\rho} F_{\rho \nu},
\end{gathered}
$$

where we have ignored any gauge fixing or ghost terms in $T_{\mu \nu}^{A}$ [39]. Since we have ignored all other interactions, all stress-energy tensors are necessarily just quadratic in matter fields.

In a QG theory we add a hat to both $T_{\mu \nu}$ and $h_{\mu \nu}$. This then results in an interaction Hamiltonian that is cubic in field operators. For example, for a complex scalar field we have terms of the form $\hat{\phi}^{\dagger} \hat{\phi} \hat{h}_{\mu \nu}$, where we have suppressed any derivatives. On the other hand, for a CG theory, the interaction Hamiltonian contains terms only quadratic in quantum field operators. For example, in the semiclassical theory of gravity $[15,17]$, with complex scalar matter fields, we have terms of the form $\hat{\phi}^{\dagger} \hat{\phi} h_{\mu \nu}$, where $h_{\mu \nu}$ is given by the expectation value of the right-hand side of Eq. (A9). Therefore, this weak-field limit of CG cannot produce or change non-Gaussianity in the state of matter, whereas QG can, as expected from the general discussion of GR and QG in the previous section.

\section{Newtonian limit}

We now consider a Newtonian theory of gravity with matter quantized. This can be obtained by starting from Newton's theory and quantizing matter or from taking the nonrelativistic limit of the above weak-field theories. For the latter, we consider a closed system and only the components $T_{00}$ and $h_{00}$. This results in Poisson's equation:

$$
\begin{gathered}
\nabla^{2} \Phi(\boldsymbol{r})=4 \pi G \rho(\boldsymbol{r}) \\
\Longrightarrow \Phi(\boldsymbol{r})=-G \int d^{3} \boldsymbol{r}^{\prime} \frac{\rho\left(\boldsymbol{r}^{\prime}\right)}{\left|\boldsymbol{r}-\boldsymbol{r}^{\prime}\right|},
\end{gathered}
$$

and the Newtonian interaction Hamiltonian

$$
H_{\mathrm{int}}=\frac{1}{2} \int d^{3} \boldsymbol{r} \rho(\boldsymbol{r}) \Phi(\boldsymbol{r}),
$$


where $\Phi:=-c^{2} h_{00} / 2$ is the Newtonian potential and $\rho:=$ $T_{00} / c^{2}$ is the matter density. Irrespective of the spin of the field, $\rho$ again contains two copies of the matter field (e.g., for a single nonrelativistic scalar matter field $\Psi, \rho=$ $\left.m \Psi^{*} \Psi\right)$. The interaction Hamiltonians for quantum and classical Newtonian gravity (with quantized scalar matter fields) are then

$$
\begin{aligned}
\hat{H}_{\mathrm{QG}}^{\mathrm{int}} & =\frac{1}{2} m \int d^{3} \boldsymbol{r}: \hat{\Psi}^{\dagger}(\boldsymbol{r}) \hat{\Psi}(\boldsymbol{r}) \hat{\Phi}(\boldsymbol{r}): \\
& =-\frac{1}{2} G m^{2} \int d^{3} \boldsymbol{r}^{\prime} d^{3} \boldsymbol{r} \frac{\hat{\Psi}^{\dagger}\left(\boldsymbol{r}^{\prime}\right) \hat{\Psi}^{\dagger}(\boldsymbol{r}) \hat{\Psi}\left(\boldsymbol{r}^{\prime}\right) \hat{\Psi}(\boldsymbol{r})}{\left|\boldsymbol{r}-\boldsymbol{r}^{\prime}\right|}
\end{aligned}
$$

$$
\hat{H}_{\mathrm{CG}}^{\mathrm{int}}=m \int d^{3} \boldsymbol{r} \hat{\Psi}^{\dagger}(\boldsymbol{r}) \hat{\Psi}(\boldsymbol{r}) \Phi[\Psi](t, \boldsymbol{r}),
$$

where :: refers to normal ordering, and we have made explicit that $\Phi$ may depend on the quantum state of matter $\Psi$ in a CG theory, which can result in singleparticle gravitational self-interaction, for which we have dropped a factor of $1 / 2$. For example, for the SchrödingerNewton equations (the nonrelativistic limit of semiclassical gravity), $\Phi$ is given by the expectation value of the right-hand side of the quantized version of Eq. (A15). Expanding the nonrelativistic field in annihilation operators, $\hat{\Psi}(\boldsymbol{r})=\sum_{k} \psi_{k}(\boldsymbol{r}) \hat{a}_{k}$, we again find CG is quadratic only in quantum operators and so cannot change the degree of quantum non-Gaussianity in the state of matter, whereas QG can.

\section{a. First quantization}

The interaction Hamiltonian of classical Newtonian gravity is given by Eq. (4). The Hamiltonian of QG and CG in the Newtonian limit can then be derived by quantizing the matter density $\rho(\boldsymbol{r})$ and, in the QG case, the gravitational potential $\Phi(\boldsymbol{r})$. In the previous section we took matter to obey a nonrelativistic quantum field $\hat{\Psi}$, such that $\hat{\rho}=m \hat{\Psi}^{\dagger} \hat{\Psi}$, assuming a single type of matter. Since $\hat{\Psi}$ is linear in annihilation operators, and so also in quadratures, the interaction Hamiltonian for CG is at most quadratic, such that an initial Gaussian state of the matter field will always remain Gaussian. However, in the case that we always have a definite particle number, which can be possible only in the Newtonian approximation of the respective theories not in the fully relativistic theories, we could also view QG and CG in a first-quantized form [225]. In this case, assuming a single type of particle, we may quantize $\rho(\boldsymbol{r})$ through

$$
\hat{\rho}(\boldsymbol{r})=m \sum_{i=1}^{N} \delta^{(3)}\left(\boldsymbol{r}-\hat{\boldsymbol{r}}_{i}\right)
$$

where $N$ is the total number of particles in the matter system. The respective QG and CG Hamiltonians would then be [226]

$$
\begin{gathered}
\hat{H}_{\mathrm{QG}}^{\mathrm{int}}=\frac{1}{2} m \sum_{i=1}^{N} \hat{\Phi}\left(\hat{\boldsymbol{r}}_{i}\right), \\
\hat{H}_{\mathrm{CG}}^{\mathrm{int}}=m \sum_{i=1}^{N} \Phi[\Psi]\left(\hat{\boldsymbol{r}}_{i}\right) .
\end{gathered}
$$

Since $\Phi(\boldsymbol{r})$ does not need to be a quadratic function of $\boldsymbol{r}$, it is possible here for CG to create non-Gaussianity in the first-quantization picture. For example, in the ShrödingerNewton equations, where $\Phi(\boldsymbol{r})=\langle\hat{\Phi}(\boldsymbol{r})\rangle$ with $\hat{\Phi}(\boldsymbol{r})$ obeying Poisson's equation (A14), the many-body wave function of $N$ massive particles would evolve as [227]

$i \hbar \partial_{t} \psi_{N}\left(t ; \boldsymbol{r}_{1}, \ldots, \boldsymbol{r}_{N}\right)=\left[-\frac{\hbar^{2}}{2 m} \sum_{i=1}^{N} \nabla_{i}^{2}+V\left(\boldsymbol{r}_{1}, \ldots, \boldsymbol{r}_{N}\right)-G m^{2} \sum_{i, j=1}^{N} \int d^{3} \boldsymbol{r}_{1}^{\prime} \cdots d^{3} \boldsymbol{r}_{N}^{\prime} \frac{\left|\psi_{N}\left(t ; \boldsymbol{r}_{1}^{\prime}, \ldots, \boldsymbol{r}_{N}^{\prime}\right)\right|^{2}}{\left|\boldsymbol{r}_{i}-\boldsymbol{r}_{j}^{\prime}\right|}\right] \psi_{N}\left(t ; \boldsymbol{r}_{1}, \ldots, \boldsymbol{r}_{N}\right)$

where $V$ is a trapping potential. Although a Gaussian approximation is expected to be very good for tabletop experiments [144,228], the evolution of $\psi_{N}$ (and hence its corresponding Wigner function) can, in principle, be non-Gaussian. Therefore, in the BEC experiment proposed in the main text, although the state of the BEC in the second-quantization picture must stay Gaussian under CG, its many-body wave function need not. Interestingly, just as particles tend to get automatically "entangled" in the first-quantization picture when we have identical particles, the particle system also tends to become automatically non-Gaussian. That is, if we have two identical particles at positions $\boldsymbol{r}_{1}$ and $\boldsymbol{r}_{2}$ and two different states $a$ and $b$, then the many-body wave function is $\psi_{N}=\left[\phi_{a}\left(\boldsymbol{r}_{1}\right) \phi_{b}\left(\boldsymbol{r}_{2}\right) \pm\right.$ 
$\left.\phi_{a}\left(\boldsymbol{r}_{2}\right) \phi_{b}\left(\boldsymbol{r}_{1}\right)\right] / \sqrt{2}$, depending on whether the particles are bosons or fermions. The system looks entangled just because of the exchange symmetry of the identical particles (it is so-called particle entangled). Similarly, even if each single-particle wave function $\phi_{a}$ and $\phi_{b}$ is Gaussian, $\psi_{N}$ will, in general, be non-Gaussian due to the exchange symmetry (and the corresponding Wigner function will be non-Gaussian also [229]). However, there has been much discussion on whether this "particle" entanglement is really physical [230-235].

\section{Quantum and classical gravity in a single BEC}

Using the Newtonian limit of gravity, the QG and CG interaction Hamiltonians for a BEC are given by Eqs. (5) and (6), with $\hat{\Psi}(\boldsymbol{r})$ representing the field of the BEC. Taking the limit of zero temperature as in the main text and ignoring any explicit time dependence of the density of the trapped BEC due to gravity, we can set $\hat{\Psi}(\boldsymbol{r})=\psi(\boldsymbol{r}) \hat{a}$, where $\psi(\boldsymbol{r})$ is the condensate wave function and $\hat{a}$ is its annihilation operator. This then results in Eqs. (8) and (9) used in the main text for the interaction Hamiltonians of QG and CG in a single BEC.

\section{APPENDIX B: EXPERIMENTAL DETAILS OF THE BOSE-EINSTEIN CONDENSATE TEST}

\section{Creating the nonclassical initial states}

In Sec. III, we describe how to create an initial singlemode squeezed state of a BEC using a spin-1 BEC and then look for non-Gaussianity. Another option would be to create a single-mode cat state and look for changes in nonGaussianity. Here we describe how such a state could be created. If we approximate the quantum field of a Bose gas by $\hat{\Psi}=\psi(\boldsymbol{r}) \hat{a}$, where $\psi$ is the condensate wave function and $\hat{a}$ is the annihilation operator for the condensate, the Hamiltonian for the electromagnetic interactions between the atoms is

$$
\hat{H}=\hbar \kappa \hat{a}^{\dagger} \hat{a}^{\dagger} \hat{a} \hat{a},
$$

where $\kappa:=\lambda_{s} /(2 \hbar)$ and $\lambda_{s}$ is defined in Eq. (21). This Hamiltonian is the Kerr interaction of quantum optics, which has been considered in BECs (see, e.g., Refs. [144, 145]). It is known that this Hamiltonian can, in principle, create a Yurke-Stoler state $|\psi\rangle=(|\alpha\rangle+i|-\alpha\rangle) / \sqrt{2}$ from an initial coherent state $|\alpha\rangle$ [123]. The evolution of such a state under QG in a BEC is considered in Appendix C.

\section{Measuring non-Gaussianity}

As described in Sec. III, measuring quadrature nonGaussianity with homodyne or heterodyne detection requires single-atom detection in a quantum gas with high efficiency on small length scales. Recent advances have opened up three promising approaches to this:

(a) After the interaction time $t$, the atomic evolution can be frozen by quickly ramping up a far-detuned optical lattice that confines atoms with a spatial resolution of the lattice wavelength, after which fluorescence-imaging light emitted by the atoms on exposure to near-resonant light fields can be detected to achieve single-atom, highspatial-resolution imaging. Single-atom-resolved imaging of a quantum gas in a two-dimensional optical lattice with submicrometer lattice spacing was first demonstrated in Refs. [236-238].

(b) A related optical fluorescence technique follows a similar working principle measuring the transit of single atoms through a light sheet that is located below the atomic sample. While the atoms are falling through the light sheet, a CCD camera records the fluorescence traces. This has been used to measure Hanbury Brown and Twiss correlations across the Bose-Einstein condensation threshold [239].

(c) Alternatively, a high-finesse cavity can be used where the transit of single atoms through the cavity will cause detectable shifts in the cavity resonance. While this technique does not allow the detection of individual atoms, the photons emerging from the cavity can be used to probe the system, revealing atom number fluctuations in real time [240,241]. Such techniques have been used to demonstrate many-body entanglement [150,242].

\section{APPENDIX C: FOURTH-ORDER CUMULANT FOR A SINGLE-MODE BOSONIC SYSTEM}

The fourth-order cumulant $k_{4}$ is given by Eq. (12) for the generalized quadrature $\hat{q}=\hat{a} e^{-i \varphi}+\hat{a}^{\dagger} e^{i \varphi}$. This requires the determination of various expectation values of combinations of $\hat{a}$ and $\hat{a}^{\dagger}$ :

$$
\begin{aligned}
\left\langle\hat{q}^{4}\right\rangle= & \frac{1}{4}\left(3+\left\langle\hat{a}^{4}\right\rangle e^{-4 i \varphi}+4\left\langle\hat{a}^{\dagger} \hat{a}^{3}\right\rangle e^{-2 i \varphi}+6\left\langle\hat{a}^{2}\right\rangle e^{-2 i \varphi}\right. \\
& \left.+12\left\langle\hat{a}^{\dagger} \hat{a}\right\rangle+6\left\langle\hat{a}^{\dagger} \hat{a}^{2}\right\rangle+\text { h.c. }\right) .
\end{aligned}
$$

The QG Hamiltonian for a single-mode BEC with electromagnetic interatomic interactions ignored is given by (8)

$$
\hat{H}_{\mathrm{QG}}=\hbar \omega \hat{a}^{\dagger} \hat{a}+\frac{1}{2} \lambda_{\mathrm{QG}} \hat{a}^{\dagger} \hat{a}^{\dagger} \hat{a} \hat{a}
$$

where we have also included the free Hamiltonian term $\hbar \omega \hat{a}^{\dagger} \hat{a}$, which derives from the kinetic and (timeindependent) trapping potential terms of the BEC Hamiltonian; see Eq. (19). Working in the Heisenberg picture, we 
find the evolution of $\hat{a}$ is

$$
\begin{aligned}
\frac{d \hat{a}(t)}{d t} & =-\frac{i}{\hbar}[\hat{a}, \hat{H}] \\
& =-i(\omega-\chi \hat{N}) \hat{a}(t),
\end{aligned}
$$

where $\hat{N}:=\hat{a}^{\dagger} \hat{a}$ and $\chi:=\left|\lambda_{\mathrm{QG}}\right| / \hbar$. Since $\hat{N}$ is a constant of motion, this can be solved as

$$
\hat{a}(t)=e^{-i \omega t} e^{i \chi \hat{N} t} \hat{a},
$$

where $\hat{a}:=\hat{a}(t=0)$. From now on we ignore the phase $\omega$ of the free evolution since this can be absorbed into the angle $\varphi$ of the quadrature $\hat{q}(\varphi)=\hat{a} e^{-i \varphi}+\hat{a}^{\dagger} e^{i \varphi}$. Then $\hat{a}^{n}$ evolves as

$$
\hat{a}^{n}(t)=e^{i(n / 2)(n-1) \chi t} e^{i n \chi \hat{N} t} \hat{a}^{n},
$$

and therefore

$$
\hat{a}^{\dagger m} \hat{a}^{n}(t)=e^{i \frac{1}{2}(n-m)(m+n-1) \chi} \hat{a}^{\dagger m} e^{i(n-m) \chi \hat{N} t} \hat{a}^{n},
$$

or equivalently

$$
\hat{a}^{\dagger m} \hat{a}^{n}(t)=e^{i \frac{1}{2}(n-m)(m-n+1) \chi t} e^{i(n-m) \chi \hat{N} t} \hat{a}^{\dagger m} \hat{a}^{n} .
$$

We can now assume $4 \chi N t \ll 1$, with $N:=\langle\hat{N}\rangle$, and expand the exponentials in Eq. (C1), that is, take

$$
e^{i n \chi \hat{N} t}=1+\operatorname{in} \chi \hat{N} t+\frac{1}{2 !} n^{2} \chi^{2} \hat{N}^{2} t^{2}+\cdots,
$$

to calculate the expectation value of $\hat{a}^{n}$, etc. In this case, if we take an initial squeezed coherent state $|\xi, \alpha\rangle$ (which is a general pure Gaussian state), $\kappa_{4}$ initially vanishes and remains zero if CG acts [see Eq. (9)], whereas under QG [see Eqs. (8) and (C2)], $\kappa_{4}$ evolves as

$$
\begin{aligned}
\kappa_{4}(t)= & -3 \chi t \sin v \sinh ^{2}(2 r) \eta_{1}(r, v)+\frac{3}{8} \chi^{2} t^{2}\left\{\sinh ^{2}(2 r) \eta_{2}(r, v)+2|\alpha|^{2}\left[2 \sinh ^{2}(2 r) \eta_{3}(r, v)+2 \sinh (2 r) \eta_{4}(r, v)\right.\right. \\
& +8 \sinh 4 r \cos 2 v \cos \psi-5 \sinh 6 r \sin 2 v \sin \psi]\}+\cdots
\end{aligned}
$$

where

$$
\begin{aligned}
\xi & :=r e^{i \vartheta}, \\
v & :=2 \varphi-\vartheta, \\
\eta_{1}(r, v) & :=\sinh 2 r-\cos v \cosh 2 r, \\
\eta_{2}(r, v) & :=6 \sinh ^{2} 2 r+8 \cos v \sinh 2 r(5 \cosh 2 r-2)-\cos 2 v(23 \cosh 4 r-16 \cosh 2 r+9), \\
\eta_{3}(r, v) & :=2 \sinh 4 r[\cos \psi(8 \cos 2 v-3)+5 \cos v]+3 \cos \psi \cos v-\cos 2 v, \\
\eta_{4}(r, v) & :=\sinh 6 r(3-8 \cos 2 v-5 \cos v \cos \psi)-\sin v \sin \psi(\cos v-10 \sinh 4 r) .
\end{aligned}
$$

In the limit of a coherent state and $N \gg 1$, we obtain the same scaling found in Ref. [91] at $\chi^{4}$ with $\varphi=\pi / 2$, whereas in the opposite limit of full squeezing, $\kappa_{4}$ tends to $24 \chi t N^{3}$ when $v=\pi / 2$, illustrating that the small value of $\chi$ can be compensated for by a large number of atoms.

If, on the other hand, we had chosen an initial Yurke-Stoler state, $|\psi\rangle:=(|\alpha\rangle+i|-\alpha\rangle) / \sqrt{2}$, then $\kappa_{4}$ at time $t$ is

$$
\kappa_{4}(t)=-8|\alpha|^{4}\left(\cos ^{4} \varphi+3 \sin ^{4} \varphi e^{-8|\alpha|^{2}}\right)-16 \chi t|\alpha|^{6} \sin 2 \varphi\left\{\cos ^{2} \varphi-e^{-4|\alpha|^{2}}\left[3+\sin ^{2} \varphi\left(2-3 e^{-4|\alpha|^{2}}\right)\right]\right\}+\cdots .
$$

In the limit $N \gg 1$, the first-order term scales as $6 \sqrt{3} \chi t N^{3}$ at $\varphi=\pi / 6$, similarly to when the initial state is $|\xi\rangle$ as above. 


\section{Nonperturbative approach}

We now pursue a nonperturbative approach to how $\kappa_{4}$ evolves with time. For the Yurke-Stoler state $|\psi\rangle:=$ $(|\alpha\rangle+i|-\alpha\rangle) / \sqrt{2}$, we can use

$$
\begin{aligned}
\left\langle\alpha\left|e^{i n \chi \hat{N} t}\right| \alpha\right\rangle & \equiv\left\langle\alpha\left|: e^{[\cos (n \chi t)+i \sin (n \chi t)-1] \hat{N}}:\right| \alpha\right\rangle \\
& =e^{[\cos (n \chi t)+i \sin (n \chi t)-1]|\alpha|^{2}}
\end{aligned}
$$

and Eqs. (C5) and (C6). For a squeezed coherent state $|\xi, \alpha\rangle$, with $\xi:=r e^{i \vartheta}$, we can use Eqs. (C5) and (C7) with

$$
\left\langle\alpha, \xi\left|e^{i n \chi \hat{N} t}\right| \xi, \alpha\right\rangle \equiv \frac{1}{\sqrt{z}} e^{\frac{1}{2} i n \chi t} G_{0}\left\langle 0\left|\hat{G}_{+} \hat{G}_{2+} \hat{G}_{3} \hat{G}_{2-} \hat{G}_{-}\right| 0\right\rangle,
$$

where

$$
\begin{aligned}
G_{0} & :=\exp \left(\beta|\alpha|^{2}-\frac{1}{2} \Lambda_{+} \alpha^{* 2}-\frac{1}{2} \Lambda_{-} \alpha^{2}\right), \\
\hat{G}_{+} & :=\exp \left[\left(\beta \alpha-\Lambda_{+} \alpha^{*}\right) \hat{a}\right], \\
\hat{G}_{-} & :=\exp \left[\left(\beta \alpha^{*}-\Lambda_{-} \alpha\right) \hat{a}^{\dagger}\right], \\
\hat{G}_{2+} & :=\exp \left(-\frac{1}{2} \Lambda_{+} \alpha^{* 2} \hat{a}^{\dagger 2}\right), \\
\hat{G}_{2-} & :=\exp \left(-\frac{1}{2} \Lambda_{-} \alpha^{2} \hat{a}^{2}\right), \\
\hat{G}_{3} & :=: \exp \beta \hat{a}^{\dagger} \hat{a}: \\
\beta & :=(1-z) / z \\
\Lambda_{+} & :=i \sinh 2 r \sin (n \chi t) e^{i \vartheta} / z, \\
\Lambda_{+} & :=i \sinh 2 r \sin (n \chi t) e^{-i \vartheta} / z, \\
z & :=\cos n \chi t-i \cosh 2 r \sin n \chi t .
\end{aligned}
$$

Here we have used the identities $\exp [\theta(A+B)] \equiv$ $\exp \theta B \exp \left[\left(e^{\theta}-1\right) A\right] \equiv \exp \left[\left(1-e^{-\theta}\right) A\right] \exp \theta B \quad$ when $[A, B]=A$, as well as [243]

$$
\begin{aligned}
& \exp \left(\gamma_{+} \hat{K}_{+}+\gamma_{-} \hat{K}_{-}+\gamma_{3} \hat{K}_{3}\right) \\
& \quad=\exp \left(\Gamma_{+} \hat{K}_{+}\right) \exp \left[\left(\ln \Gamma_{3}\right) \hat{K}_{3}\right] \exp \left(\Gamma_{-} \hat{K}_{-}\right),
\end{aligned}
$$

with

$$
\begin{gathered}
\Gamma_{3}=\left(\cosh \beta-\frac{\gamma_{3}}{2 \beta} \sinh \beta\right)^{-2}, \\
\Gamma_{ \pm}=\frac{2 \gamma_{ \pm} \sinh \beta}{2 \beta \cosh \beta-\gamma_{3} \sinh \beta} \\
\beta^{2}=\frac{1}{4} \gamma_{3}^{2}-\gamma_{+} \gamma_{-},
\end{gathered}
$$

and $\left[\hat{K}_{3}, \hat{K}_{ \pm}\right]= \pm \hat{K}_{ \pm}$and $\left[\hat{K}_{+}, \hat{K}_{-}\right]=-2 \hat{K}_{3}$. For example, with use of Eq. $(\mathrm{C} 17),\left\langle\xi\left|\hat{a}^{4}(t)\right| \xi\right\rangle$ under $\hat{H}_{\mathrm{QG}}$ can be shown to be

$$
\left\langle\xi\left|\hat{a}^{4}(t)\right| \xi\right\rangle=\frac{3 e^{-4 i \chi t+2 i \vartheta} \sinh ^{2} 2 r}{2^{2}(\cos 4 \chi t-i \cosh 2 r \sin 4 \chi t)^{5 / 2}},
$$

where we can use $\sqrt{z} \equiv \sqrt{|z|}(z+|z|) /|z+| z||$ to remove the square root of the complex number.

\section{Including the reverse process}

Above we consider the evolution of $\kappa_{4}$ under the QG Hamiltonian $\hat{H}_{\mathrm{QG}}$ and assuming that the BEC is prepared in either a squeezed coherent state or a Yurke-Stoler state. In the main text, we also consider a measurement protocol where we first prepare the BEC state that is required, let the BEC evolve under QG, and then measure $\kappa_{4}$ after we have applied the reverse process to that we used to create the initial BEC state. In the Heisenberg picture, $\hat{a}$ then undergoes the following evolutions:

(a) $\hat{a} \rightarrow \hat{a}^{\prime}=\hat{U}_{\Psi}^{\dagger} \hat{a} \hat{U}_{\Psi} \quad$ at $\quad t=0$.

(b) $\hat{a} \rightarrow \hat{a}^{\prime \prime}(t)=e^{i \chi \hat{N} t} \hat{a}^{\prime}$ for $0<t<\tau$.

(c) $\hat{a} \rightarrow \hat{a}^{\prime \prime \prime}(\tau)=\hat{U}_{-\Psi}^{\dagger} \hat{a}_{2}(\tau) \hat{U}_{-\Psi}$ at $t=\tau$.

Hare $\hat{U}_{\Psi}$ refers to the unitary that creates the initial state and $\hat{U}_{-\Psi}$ is the reverse process. For example, if a squeezed vacuum state is prepared, then $\hat{U}_{\Psi}=\exp \left[r\left(e^{i \vartheta} \hat{a}^{\dagger 2}-\right.\right.$ $\left.\left.e^{-i \vartheta} \hat{a}^{2}\right) / 2\right]$ and $\hat{U}_{-\Psi}=\exp \left[-r\left(e^{i \vartheta} \hat{a}^{\dagger 2}-e^{-i \vartheta} \hat{a}^{2}\right) / 2\right]$. In this case, in the limit that $\chi \ll 1, \kappa_{4}$ at the end of the process is given by

$$
\kappa_{4}(\tau)=\frac{3}{2} \chi \tau \sin 2 v \sinh (2 r)^{2}+\cdots
$$

with $v$ given by Eq. (C11). In the limit of large $N$, this scales as $\chi \tau N^{2}$ in contrast to the $N^{3}$ scaling for the process considered previously; see Eq. (C9). However, the SNR scaling is the same.

\section{APPENDIX D: ESTIMATION OF THE FOURTH-ORDER CUMULANT SIGNAL-TO-NOISE RATIO}

The SNR for measuring the fourth-order cumulant $\kappa_{4}$ is given by

$$
\mathrm{SNR}=\frac{\left|\kappa_{4}\right|}{\sqrt{\operatorname{var} k_{4}}},
$$


where $k_{4}$ is the fourth $k$ statistic. The variance of $k_{4}$ is given by [126]

$$
\begin{aligned}
\operatorname{var} k_{4}= & \frac{\kappa_{8}}{\mathcal{M}}+\frac{16 \kappa_{2} \kappa_{6}}{\mathcal{M}-1}+\frac{48 \kappa_{3} \kappa_{5}}{\mathcal{M}-1}+\frac{34 \kappa_{4}^{2}}{\mathcal{M}-1} \\
& +\frac{72 \mathcal{M} \kappa_{2}^{2} \kappa_{4}}{(\mathcal{M}-1)(\mathcal{M}-2)}+\frac{144 \mathcal{M} \kappa_{2} \kappa_{3}^{2}}{(\mathcal{M}-1)(\mathcal{M}-2)} \\
& +\frac{24 \mathcal{M}(\mathcal{M}+1) \kappa_{2}^{4}}{(\mathcal{M}-1)(\mathcal{M}-2)(\mathcal{M}-3)},
\end{aligned}
$$

where $\mathcal{M}$ is the number of independent estimations. In the limit $\mathcal{M} \gg 1$, var $k_{4}$ becomes

$$
\begin{aligned}
\operatorname{var} k_{4} \approx & \frac{1}{\mathcal{M}}\left(\kappa_{8}+16 \kappa_{2} \kappa_{6}+48 \kappa_{3} \kappa_{5}+34 \kappa_{4}^{2}+72 \kappa_{2}^{2} \kappa_{4}\right. \\
& \left.+144 \kappa_{2} \kappa_{3}^{2}+24 \kappa_{2}^{4}\right) .
\end{aligned}
$$

The $n$ th-order cumulant $\kappa_{n}$ can be found using

$$
\kappa_{n}=\mu_{n}-\sum_{m=1}^{n-1}\left(\begin{array}{c}
n-1 \\
m-1
\end{array}\right) \mu_{n-m} \kappa_{m},
$$

where $\mu_{n}:=\left\langle\hat{q}^{n}\right\rangle$ is the $n$th moment.

Using results from Appendix $C$, in the limit that $\chi \ll 1$, the SNR for the estimation of $\kappa_{4}$ for a squeezed vacuum state $|\xi\rangle$ is given by

$$
\begin{aligned}
& \sqrt{6 \mathcal{M}} t \chi \sinh ^{2} 2 r \frac{|\sin v(\sinh 2 r-\cos v \cosh 2 r)|}{(\cosh 2 r-\cos v \sinh 2 r)^{2}} \\
& +\cdots
\end{aligned}
$$

This is maximized at the angles

$$
\varphi=\frac{1}{2}\left(\vartheta \pm \frac{1}{2} \cos ^{-1} y\right)
$$

where

$$
y:=\frac{\sinh ^{2} 2 r\left(\sinh ^{2} 2 r-2\right) \pm 2 \sqrt{2} \sinh 4 r}{\left(\sinh ^{2} 2 r+2\right)^{2}},
$$

which results in the above SNR being approximately $4.9 \chi t N^{2} \sqrt{\mathcal{M}}$ for $N \gg 1$. When $\chi N^{2} t$ is not small, this SNR approximation is not so accurate, and instead the results of the previous section can be used to find a nonperturbative solution for the SNR. For example, for the BMV proposal values $d=200 \mu \mathrm{m}, t=2 \mathrm{~s}$, and $M=10^{-14} \mathrm{~kg}$, we find that the maximum SNR for a spherical ${ }^{133} \mathrm{Cs}$ BEC is approximately $0.3 \sqrt{\mathcal{M}}$ (with the value of $d$ being used for the radius $R$ ). At these values, $\chi N^{2} t=\sqrt{2 / \pi} \phi \approx 0.5$, where $\phi=0.6$ is the relative phase expected in the BMV experiment when all distances between the microspheres other than $d$, the smallest possible distance, are ignored. Therefore, the SNR is still of order $\chi t N^{2} \sqrt{\mathcal{M}}$ in this case. If instead the mass is lowered to $M=10^{-15} \mathrm{~kg}$, then we can use the approximation that the SNR is given by $4.9 \times t N^{2} \sqrt{\mathcal{M}}$.

For the protocol where we reverse the squeezing operation before the measurement, the SNR is given by

$$
\sqrt{\frac{3}{2}} \chi \tau|\sin 2 v| \sinh ^{2} 2 r+\cdots
$$

in the limit that $\chi \ll 1$.

\section{APPENDIX E: EVOLUTION UNDER CLASSICAL GRAVITY}

Here we consider how a single BEC evolves under CG compared with QG. We start with the general Newtonian expressions (5) and (6). Working in the Schrödinger picture, we find for QG the evolution of our state vector $|\Psi\rangle$ is given by

$$
i \hbar \frac{d|\Psi(t)\rangle}{d t}=\hat{H}_{\mathrm{QG}}^{\mathrm{BEC}}|\Psi(t)\rangle
$$

where

$$
\begin{aligned}
\hat{H}_{\mathrm{QG}}^{\mathrm{BEC}}:= & \int d^{3} \boldsymbol{r}\left[-\frac{\hbar^{2}}{2 m} \hat{\Psi}^{\dagger}(\boldsymbol{r}) \nabla^{2} \hat{\Psi}(\boldsymbol{r})+V(\boldsymbol{r}) \hat{\Psi}^{\dagger}(\boldsymbol{r}) \hat{\Psi}(\boldsymbol{r})\right. \\
& \left.+\frac{1}{2} m: \hat{\Psi}^{\dagger}(\boldsymbol{r}) \hat{\Psi}(\boldsymbol{r}) \hat{\Phi}(\boldsymbol{r}):\right] \\
= & \int d^{3} \boldsymbol{r}\left[-\frac{\hbar^{2}}{2 m} \hat{\Psi}^{\dagger}(\boldsymbol{r}) \nabla^{2} \hat{\Psi}(\boldsymbol{r})+V(\boldsymbol{r}) \hat{\Psi}^{\dagger}(\boldsymbol{r}) \hat{\Psi}(\boldsymbol{r})\right. \\
& \left.-\frac{1}{2} G m^{2} \int d^{3} \boldsymbol{r}^{\prime} \frac{\hat{\Psi}^{\dagger}(\boldsymbol{r}) \hat{\Psi}^{\dagger}\left(\boldsymbol{r}^{\prime}\right) \hat{\Psi}(\boldsymbol{r}) \hat{\Psi}\left(\boldsymbol{r}^{\prime}\right)}{\left|\boldsymbol{r}-\boldsymbol{r}^{\prime}\right|}\right], \quad(\mathrm{E} 2)
\end{aligned}
$$

with $V(\boldsymbol{r})$ the trapping potential. In contrast, for $\mathrm{CG}$, we have

$$
i \hbar \frac{d|\Psi(t)\rangle}{d t}=\hat{H}_{\mathrm{CG}}^{\mathrm{BEC}}[\Psi](t)|\Psi(t)\rangle
$$

where

$$
\begin{aligned}
\hat{H}_{\mathrm{CG}}^{\mathrm{BEC}}[\Psi](t) & \\
:= & \int d^{3} \boldsymbol{r}\left[-\frac{\hbar^{2}}{2 m} \hat{\Psi}^{\dagger}(\boldsymbol{r}) \nabla^{2} \hat{\Psi}(\boldsymbol{r})+V(\boldsymbol{r}) \hat{\Psi}^{\dagger}(\boldsymbol{r}) \hat{\Psi}(\boldsymbol{r})\right. \\
& \left.+m \hat{\Psi}^{\dagger}(\boldsymbol{r}) \hat{\Psi}(\boldsymbol{r}) \Phi[\Psi(t)](\boldsymbol{r})\right] .
\end{aligned}
$$


In the Schrödinger-Newton example of CG, we have

$$
\begin{aligned}
\hat{H}_{\mathrm{CG}}^{\mathrm{BEC}}[\Psi](t) & \\
= & \int d^{3} \boldsymbol{r}\left[-\frac{\hbar^{2}}{2 m} \hat{\Psi}^{\dagger}(\boldsymbol{r}) \nabla^{2} \hat{\Psi}(\boldsymbol{r})+V(\boldsymbol{r}) \hat{\Psi}^{\dagger}(\boldsymbol{r}) \hat{\Psi}(\boldsymbol{r})\right. \\
& \left.-G m^{2} \int d^{3} \boldsymbol{r}^{\prime} \frac{\hat{\Psi}^{\dagger}(\boldsymbol{r}) \hat{\Psi}(\boldsymbol{r})\left\langle\Psi(t)\left|\hat{\Psi}^{\dagger}\left(\boldsymbol{r}^{\prime}\right) \hat{\Psi}\left(\boldsymbol{r}^{\prime}\right)\right| \Psi(t)\right\rangle}{\left|\boldsymbol{r}-\boldsymbol{r}^{\prime}\right|}\right] .
\end{aligned}
$$

The evolution of $|\Psi\rangle$ in CG is, in general, "nonlinear" in that $|\Psi\rangle$ is needed to determine $\Phi$. This is often referred to as a wave function "self-interaction" since in the firstquantization picture, the wave function of a single particle will now interact with itself, something that can never occur in a quantum theory of gravity, where Eq. (E1) is said to be "linear."

If we ignore any explicit time dependence, the evolution of $|\Psi\rangle$ in QG can, in principle, be solved as

$$
|\Psi(t)\rangle=e^{-i \hat{H}_{\mathrm{QG}}^{\mathrm{BEC}} t / \hbar}|\Psi(0)\rangle .
$$

In contrast, it may not be possible to find an analytic solution in CG due to the potential nonlinearities. However, the evolution will still take the form

$$
|\Psi(t)\rangle=\hat{T}\left\{e^{-(i / \hbar) \int_{0}^{t} d \tau \hat{H}_{\mathrm{CG}}^{\mathrm{BEC}}[\Psi](\tau)}\right\}|\Psi(0)\rangle,
$$

where $\hat{T}$ is the time-ordering operator. Despite the potential nonlinearity, since $\hat{H}_{\mathrm{CG}}^{\mathrm{BEC}}$ is quadratic in matter field operators, it is still a Gaussian process. For example, consider the single-mode BEC experiment introduced in the main text where we assume $\hat{\Psi}(\boldsymbol{r})=\psi(\boldsymbol{r}) \hat{a}$. Ignoring the trapping potential and free dynamics, we have

$$
|\Psi(t)\rangle=\hat{T}\left\{e^{-(i / \hbar) \int_{0}^{t} d \tau \lambda_{\mathrm{CG}}[\Psi](t) \hat{a}^{\dagger} \hat{a}}\right\}|\Psi(0)\rangle,
$$

with

$$
\lambda_{\mathrm{CG}}[\Psi](t)=m \int d^{3} \boldsymbol{r}|\psi(\boldsymbol{r})|^{2} \Phi[\Psi](t, \boldsymbol{r})
$$

Equation (E8) can be written as [94]

$$
|\Psi(t)\rangle=e^{-(i / \hbar) \Lambda_{\mathrm{CG}}[\Psi](t) \hat{a}^{\dagger} \hat{a}}|\Psi(0)\rangle,
$$

where

$$
\Lambda_{\mathrm{CG}}[\Psi](t):=\int_{0}^{t} d \tau \lambda_{\mathrm{CG}}[\Psi](\tau)
$$

The evolution of $|\Psi\rangle$ in this case is then, in general, a nonlinear Gaussian process. However, it need not always be nonlinear. For instance, in the Schrödinger-Newton case we have

$$
\begin{aligned}
\lambda_{\mathrm{CG}}[\Psi](t)= & -G m^{2}\langle\Psi(t)|\hat{N}| \Psi(t)\rangle \\
& \times \int d^{3} \boldsymbol{r} d^{3} \boldsymbol{r}^{\prime} \frac{\left|\psi\left(\boldsymbol{r}^{\prime}\right)\right|^{2}|\psi(\boldsymbol{r})|^{2}}{\left|\boldsymbol{r}-\boldsymbol{r}^{\prime}\right|},
\end{aligned}
$$

where $\hat{N}:=\hat{a}^{\dagger} \hat{a}$. Since $\hat{N}$ is a constant of motion (it commutes with $\hat{H}_{\mathrm{CG}}^{\mathrm{BEC}}$ ), we have

$$
\lambda_{\mathrm{CG}}=-G m^{2} N \int d^{3} \boldsymbol{r} d^{3} \boldsymbol{r}^{\prime} \frac{\left|\psi\left(\boldsymbol{r}^{\prime}\right)\right|^{2}|\psi(\boldsymbol{r})|^{2}}{\left|\boldsymbol{r}-\boldsymbol{r}^{\prime}\right|},
$$

where $N:=\langle\hat{N}\rangle$. Therefore, $|\Psi(t)\rangle$ evolves as

$$
|\Psi(t)\rangle=e^{-(i / \hbar) \gamma_{\mathrm{CG}} \hat{a}^{\dagger} \hat{a} t}|\Psi(0)\rangle,
$$

where

$\gamma_{\mathrm{CG}}:=\int d^{3} \boldsymbol{r}\left[-\frac{\hbar^{2}}{2 m} \psi^{*}(\boldsymbol{r}) \nabla^{2} \psi(\boldsymbol{r})+V(\boldsymbol{r})|\psi(\boldsymbol{r})|^{2}\right]-\lambda_{\mathrm{CG}}$,

such that $|\Psi(t)\rangle$ evolves under a Gaussian phase-shift channel. For example, if the BEC were initially in a coherent state $|\alpha\rangle$, it would stay in a coherent state but with just a time-dependent phase:

$$
|\Psi(t)\rangle=\left|\alpha e^{-i \gamma_{C G} t / \hbar}\right\rangle,
$$

with $N=|\alpha|^{2}$.

\section{APPENDIX F: STOCHASTIC AND COMPLEX INTERACTIONS}

Here we consider matter interacting with a complex, stochastic nonquantum field (non-operator-valued distribution) and why this interaction cannot, in the absence of all other interactions, turn a Gaussian state into a nonGaussian state, where the latter is defined as any state that does not belong to the Gaussian convex hull [177].

In the main text, we consider interacting matter with a classical entity $\mathcal{G}$ (a quantity that takes on real and welldefined values) and how this can be distinguished from the quantum version of the interaction. If we take, for simplicity, matter to be described by a real scalar quantum field $\hat{\phi}$, then as long as we do not allow the classical interaction to induce quantum self-interactions of matter, $\mathcal{G}$ and $\hat{\phi}$ can interact only through Hamiltonian terms that are linear or quadratic in $\hat{\phi}$. That is, the Hamiltonian density of the interaction must be of the form

$$
\hat{\mathcal{H}}=s[\hat{\phi}] f[\mathcal{G}]+t[\hat{\phi}] h[\mathcal{G}],
$$

where $s$ and $t$ are, respectively, linear and quadratic real functionals of $\hat{\phi}$, and $f$ and $h$ are general real functionals of 
$\mathcal{G}$. It is shown in the main text that a Hamiltonian density of the form of Eq. (F1) preserves the Gaussianity of the matter field, and we can use this fact to distinguish it from a quantum interaction.

We now, in contrast to the main text, allow $\mathcal{G}$, or $f$ and $h$, to be complex valued. Expanding $\hat{\phi}$ in creation and annihilation operators, $\hat{\phi}=\sum_{k}\left[u_{k}(t) \hat{a}_{k}+v_{k}(t) \hat{a}_{k}^{\dagger}\right]$, we find the corresponding Hamiltonian will be of the form of Eq. (2) except that now $\boldsymbol{\lambda}_{k}(t), \boldsymbol{\mu}_{k l}(t) \in \mathbb{C}$, so the Hamiltonian is, in general, non-Hermitian. Despite this, the quadratic nature of the non-Hermitian Hamiltonian means that it still preserves the Gaussian form of the Wigner function for an initial Gaussian state [171-174]. For example, consider the Hamiltonian $\hat{H}=\lambda \hat{a}^{\dagger} \hat{a}$, where $\lambda:=\lambda_{R}-i \lambda_{I}$. Under this Hamiltonian, an initial coherent state $|\alpha\rangle$ will evolve to $\exp \left\{-|\alpha|^{2}\left[1-\exp \left(-2 \lambda_{I} t\right)\right] / 2\right\}|\alpha \exp (-i \lambda t)\rangle$, which is just an unnormalized, damped coherent state with a timedependent phase (note that we have taken $\hbar=1$ here and do so throughout the rest of this appendix). In general, a non-Hermitian Hamiltonian will lead to an unnormalized state. To rectify this, the physical state vector can be defined as $\left.\left|\psi^{\mathcal{N}}\right\rangle:=|\psi\rangle / \| \psi\right\rangle \mid$. For the above example, this would mean that an initial coherent state evolves to a damped coherent state with a time-dependent phase: $\left|\alpha^{\prime}(t) \exp \left(-\lambda_{I} t\right)\right\rangle$, where $\alpha^{\prime}(t):=\alpha \exp \left(-i \lambda_{R} t\right)$.

We now take $\mathcal{G}$ to be a stochastic field, which we denote as $\tilde{\mathcal{G}}$, and keep $f$ and $h$ complex valued. The interaction Hamiltonian density (F1) can then be written as

$$
\hat{\mathcal{H}}[\tilde{\mathcal{G}}]=s[\hat{\phi}] f[\tilde{\mathcal{G}}]+t[\hat{\phi}] h[\tilde{\mathcal{G}}] .
$$

In the interaction picture, an out state $\left|\psi_{\text {out }}[\tilde{\mathcal{G}}]\right\rangle$ of the quantum field $\hat{\phi}$ is now given by a stochastic $S$ matrix $\hat{S}[\tilde{\mathcal{G}}]$ acting on the in state $\left|\psi_{\text {in }}\right\rangle$ [182]. That is,

$$
\left|\psi_{\text {out }}[\tilde{\mathcal{G}}]\right\rangle=\hat{S}[\tilde{\mathcal{G}}]\left|\psi_{\text {in }}\right\rangle
$$

where

$$
\hat{S}[\tilde{\mathcal{G}}]:=T e^{-i \int d^{4} x\left(\hat{\mathcal{H}}_{0}+\hat{\mathcal{H}}[\tilde{\mathcal{G}}]\right)},
$$

with $T$ the time-ordering operator, $x$ a four coordinate, and $\hat{\mathcal{H}}_{0}$ the free (nonstochastic) Hamiltonian density.

Since the Hamiltonian may not be Hermitian, the out state may not be normalized, but we can define a normalized out state as

$$
\left|\psi_{\text {out }}^{\mathcal{N}}[\tilde{\mathcal{G}}]\right\rangle:=\mathcal{N}^{-1 / 2}\left|\psi_{\text {out }}[\tilde{\mathcal{G}}]\right\rangle
$$

where

$$
\mathcal{N}:=\left\langle\psi_{\text {out }}[\tilde{\mathcal{G}}] \mid \psi_{\text {out }}[\tilde{\mathcal{G}}]\right\rangle
$$

The density matrix corresponding to a particular out state $\left|\psi_{\text {out }}[\tilde{\mathcal{G}}]\right\rangle$ can be defined as usual,

$$
\hat{\rho}_{\text {out }}[\tilde{\mathcal{G}}]:=\left|\psi_{\text {out }}[\tilde{\mathcal{G}}]\right\rangle\left\langle\psi_{\text {out }}[\tilde{\mathcal{G}}]\right|,
$$

or as the normalized version,

$$
\hat{\rho}_{\text {out }}^{\mathcal{N}}[\tilde{\mathcal{G}}]:=\mathcal{N}^{-1} \hat{\rho}_{\text {out }}[\tilde{\mathcal{G}}] .
$$

From Eq. (F3), the density matrix $\hat{\rho}_{\text {out }}[\tilde{\mathcal{G}}]:=\left|\psi_{\text {out }}[\tilde{\mathcal{G}}]\right\rangle$ $\left\langle\psi_{\text {out }}[\tilde{\mathcal{G}}]\right|$ can be found through

$$
\hat{\rho}_{\text {out }}[\tilde{\mathcal{G}}]=\hat{S}[\tilde{\mathcal{G}}] \hat{\rho}_{\text {in }} \hat{S}^{\dagger}[\tilde{\mathcal{G}}],
$$

where $\hat{\rho}_{\text {in }}:=\left|\psi_{\text {in }}\right\rangle\left\langle\psi_{\text {in }}\right|$. The above density matrix corresponds to a particular stochastic out state $\left|\psi_{\text {out }}[\tilde{\mathcal{G}}]\right\rangle$. However, the quantity that provides the correct expectation values of operators $\left[\langle\hat{A}\rangle=\operatorname{Tr}\left(\hat{\rho}_{\text {out }} \hat{A}\right)\right]$ is the average density matrix (averaged over $\tilde{\mathcal{G}}) \hat{\rho}_{\text {out }}[244,245]$. That is, $\hat{\rho}_{\text {out }}$ is given by [182]

$$
\begin{aligned}
\hat{\rho}_{\text {out }} & :=\int \mathcal{D} \tilde{\mathcal{G}} P[\tilde{\mathcal{G}}]\left(\hat{\rho}_{\text {out }}[\tilde{\mathcal{G}}]\right) \\
& =\int \mathcal{D} \tilde{\mathcal{G}} P[\tilde{\mathcal{G}}]\left(\hat{S}[\tilde{\mathcal{G}}] \hat{\rho}_{\text {in }} \hat{S}^{\dagger}[\tilde{\mathcal{G}}]\right) \\
& :=\int \mathcal{D} \tilde{\mathcal{G}} P[\tilde{\mathcal{G}}]\left(\hat{\mathcal{S}}_{S}[\tilde{\mathcal{G}}] \hat{\rho}_{\text {in }}\right) \\
& :=\hat{\mathcal{S}}_{\text {av }} \hat{\rho}_{\text {in }},
\end{aligned}
$$

where $P[\tilde{\mathcal{G}}]$ is the probability distribution functional of $\tilde{\mathcal{G}}, \hat{\mathcal{S}}_{S}[\tilde{\mathcal{G}}]$ is the scattering superoperator, and $\hat{\rho}_{\text {in }}$ now, in general, corresponds to a general initial mixed state $[179,246]$.

If we take $\hat{\rho}_{\text {in }}$ to be a pure Gaussian state, then since each $\hat{S}[\tilde{\mathcal{G}}]$ is associated with a Gaussian transformation [i.e., Eq. (F1)], Eq. (F10) is just the stochastic quantum field theory generalization of a state, $\hat{\rho}_{C H}$, in the Gaussian convex hull of quantum optics [177]:

$$
\hat{\rho}_{C H}=\int d \boldsymbol{g} P(\boldsymbol{g}) \hat{\rho}_{G}(\boldsymbol{g}),
$$

where $\boldsymbol{g}$ is a set of complex numbers, $P(\boldsymbol{g})$ is a probability distribution, and $\hat{\rho}_{G}(\boldsymbol{g})=\left|\psi_{G}(\boldsymbol{g})\right\rangle\left\langle\psi_{G}(\boldsymbol{g})\right|$ is a pure Gaussian density matrix. Defining a Gaussian state as a pure state with a Gaussian Wigner function or a mixture of pure states with Gaussian Wigner functions represents a broader definition of a Gaussian state compared with the more conventional definition of any state with a Gaussian Wigner function that is used in the main text $[92,177]$. A non-Gaussian state (also sometimes referred to as a "quantum" non-Gaussian state to distinguish it from the more 
conventional definition of a non-Gaussian state [92]) can then be defined as any state that lives outside the convex hull of Gaussian states [247].

As shown and discussed in the main text, to rule out a classical interaction (defined as an interaction with a nonquantum field that takes on real and well-defined values, such as the classical electromagnetic or gravitational fields) any detection of a non-Gaussian state as it is conventionally defined (any state with a non-Gaussian Wigner function) is sufficient as long as all other interactions can be ignored. As shown above, this also applies when the field takes on complex values. However, to rule out a stochastic interaction (defined as an interaction with a nonquantum field that is fundamentally stochastic, sometimes referred to as a "postquantum" interaction), we must appeal to the detection of a non-Gaussian state (or "quantum" non-Gaussian state) in its broader definition as any state that sits outside the Gaussian convex hull [248]. This is to be expected since a Gaussian state evolves to a state in the Gaussian convex hull if there is a combination of Gaussian operations and statistical randomization [93].

\section{Example: A stochastic and complex generalization of the Newtonian gravitational interaction}

We now consider a specific example of a stochastic and complex interaction that, when we take the nonrelativistic limit, could be considered as a stochastic and complex generalization of the Newtonian gravitational interaction. The relativistic version of this interaction has the Hamiltonian density

$$
\hat{\mathcal{H}}=\hat{A} \tilde{h}[\tilde{\mathcal{G}}]
$$

where $\hat{A}:=\hat{\phi}^{\dagger} \hat{\phi}$ is a mass-density-like operator for a complex relativistic scalar field $\hat{\phi}$ and $\tilde{h}[\tilde{\mathcal{G}}]$ is defined as

$$
\tilde{h}[\tilde{\mathcal{G}}(x)]:=\int d^{4} x^{\prime} \Lambda\left(x, x^{\prime}\right) \tilde{\mathcal{G}}\left(x^{\prime}\right),
$$

with $\Lambda\left(x, x^{\prime}\right):=\Lambda_{R}\left(x, x^{\prime}\right)-i \Lambda_{I}\left(x, x^{\prime}\right), \Lambda_{R}\left(x, x^{\prime}\right)$ a real kernel, $\Lambda_{I}\left(x, x^{\prime}\right)$ a positive definite kernel, and $\tilde{\mathcal{G}}(x)$ a real stochastic field. The stochastic (Gaussian) scattering matrix $\hat{S}[\tilde{\mathcal{G}}]$ is then (ignoring $\hat{\mathcal{H}}_{0}$ for simplicity)

$$
\begin{aligned}
\hat{S}[\tilde{\mathcal{G}}]= & T e^{-i \int d^{4} x d^{4} x^{\prime} \Lambda_{R}\left(x, x^{\prime}\right) \hat{\phi}^{\dagger}\left(x^{\prime}\right) \hat{\phi}\left(x^{\prime}\right) \tilde{\mathcal{G}}\left(x^{\prime}\right)} \\
& \times e^{-\int d^{4} x d^{4} x^{\prime} \Lambda_{I}\left(x, x^{\prime}\right) \hat{\phi}^{\dagger}\left(x^{\prime}\right) \hat{\phi}\left(x^{\prime}\right) \tilde{\mathcal{G}}\left(x^{\prime}\right)},
\end{aligned}
$$

such that the (Gaussian) stochastic scattering superoperator is

$$
\begin{aligned}
\hat{\mathcal{S}}_{S}[\tilde{\mathcal{G}}]= & \hat{T} \exp \left[-i \int d^{4} x d^{4} x^{\prime} \Lambda_{R}\left(x, x^{\prime}\right) \hat{A}_{\Delta}\left(x^{\prime}\right) \tilde{\mathcal{G}}\left(x^{\prime}\right)\right. \\
& \left.-\int d^{4} x d^{4} x^{\prime} \Lambda_{I}\left(x, x^{\prime}\right) \hat{A}_{\Sigma}\left(x^{\prime}\right) \tilde{\mathcal{G}}\left(x^{\prime}\right)\right],
\end{aligned}
$$

where $\hat{T}$ is the time-ordering superoperator, $\hat{A}_{\Delta}=\hat{A}_{+}-$ $\hat{A}_{-}$, and $\hat{A}_{\Sigma}=\hat{A}_{+}+\hat{A}_{-}$, with $\hat{A}_{+}$representing $\hat{A}$ acting on $\hat{\rho}_{\text {in }}$ from the left and $\hat{A}_{-}$representing $\hat{A}$ acting on $\hat{\rho}_{\text {in }}$ from the right. Taking, for convenience, the probability distribution functional to be Gaussian,

$$
P[\tilde{\mathcal{G}}]=(\operatorname{det} \Gamma)^{1 / 2} e^{-\int d^{4} x d^{4} x^{\prime} \Gamma\left(x, x^{\prime}\right) \tilde{\mathcal{G}}(x) \tilde{\mathcal{G}}\left(x^{\prime}\right)},
$$

with $\Gamma\left(x, x^{\prime}\right)$ a positive-definite symmetric kernel, we can perform Gaussian functional integration (F10) over $\tilde{\mathcal{G}}$ to obtain $\hat{\rho}_{\text {out }}=\hat{\mathcal{S}}_{\text {av }} \hat{\rho}_{\text {in }}$, with

$$
\hat{\mathcal{S}}_{\mathrm{av}}=\hat{T} e^{\int d^{4} x d^{4} x^{\prime}\left[-\beta_{R R}\left(x, x^{\prime}\right) \hat{A}_{\Delta}(x) \hat{A}_{\Delta}\left(x^{\prime}\right)+i \beta_{I R}\left(x, x^{\prime}\right) \hat{A}_{\Delta}(x) \hat{A}_{\Sigma}\left(x^{\prime}\right)+i \beta_{I R}\left(x, x^{\prime}\right) \hat{A}_{\Sigma}(x) \hat{A}_{\Delta}\left(x^{\prime}\right)+\beta_{I I}\left(x, x^{\prime}\right) \hat{A}_{\Sigma}(x) \hat{A}_{\Sigma}\left(x^{\prime}\right)\right]},
$$

where

$$
\begin{aligned}
& \beta_{R R}\left(x, x^{\prime}\right):=\frac{1}{4} \int d^{4} x^{\prime \prime} d^{4} x^{\prime \prime \prime} \Lambda_{R}\left(x, x^{\prime \prime}\right) \Gamma^{-1}\left(x^{\prime \prime}, x^{\prime \prime \prime}\right) \Lambda_{R}\left(x^{\prime}, x^{\prime \prime \prime}\right), \\
& \beta_{I R}\left(x, x^{\prime}\right):=\frac{1}{4} \int d^{4} x^{\prime \prime} d^{4} x^{\prime \prime \prime} \Lambda_{R}\left(x, x^{\prime \prime}\right) \Gamma^{-1}\left(x^{\prime \prime}, x^{\prime \prime \prime}\right) \Lambda_{I}\left(x^{\prime}, x^{\prime \prime \prime}\right), \\
& \beta_{I I}\left(x, x^{\prime}\right):=\frac{1}{4} \int d^{4} x^{\prime \prime} d^{4} x^{\prime \prime \prime} \Lambda_{I}\left(x, x^{\prime \prime}\right) \Gamma^{-1}\left(x^{\prime \prime}, x^{\prime \prime \prime}\right) \Lambda_{I}\left(x^{\prime}, x^{\prime \prime \prime}\right)
\end{aligned}
$$

We now take a Markovian approximation and define $\Lambda_{R}, \Lambda_{I}$, and $\Gamma$ as [182]

$$
\begin{gathered}
\Lambda_{R, I}\left(x, x^{\prime}\right)=\lambda_{R, I}\left(x_{0}, \boldsymbol{r}, \boldsymbol{r}^{\prime}\right) \delta\left(x_{0}-x_{0}^{\prime}\right), \\
\Gamma\left(x, x^{\prime}\right)=\gamma\left(x_{0}, \boldsymbol{r}, \boldsymbol{r}^{\prime}\right) \delta\left(x_{0}-x_{0}^{\prime}\right) .
\end{gathered}
$$


The superoperator $\hat{S}_{\text {av }}$ can then be written as

$$
\hat{S}_{\mathrm{av}}=\hat{T} \exp \left[\int_{-\infty}^{\infty} d t \hat{\mathcal{L}}(t)\right],
$$

where $\hat{\mathcal{L}}(t)$ is the linear evolution superoperator and $\hat{T}$ is the time-ordering superoperator [182]. The averaged density matrix $\hat{\rho}$ at time $t$ can now be obtained through

$$
\hat{\rho}(t)=\hat{T} \exp \left[\int_{0}^{t} d \tau \hat{\mathcal{L}}(\tau)\right] \hat{\rho}(0) .
$$

The superoperator $\hat{\mathcal{L}}$ acts on $\hat{\rho}$ as

$$
\hat{\mathcal{L}} \hat{\rho}=\int d \boldsymbol{r} d \boldsymbol{r}^{\prime}\left(2 i b_{I R}\left(t, \boldsymbol{r}, \boldsymbol{r}^{\prime}\right)\left[\hat{A}(t, \boldsymbol{r}) \hat{A}\left(t, \boldsymbol{r}^{\prime}\right), \hat{\rho}\right]-b_{R R}\left(t, \boldsymbol{r}, \boldsymbol{r}^{\prime}\right)\left[\hat{A}(t, \boldsymbol{r}),\left[\hat{A}\left(t, \boldsymbol{r}^{\prime}\right), \hat{\rho}\right]\right]+b_{I I}\left(t, \boldsymbol{r}, \boldsymbol{r}^{\prime}\right)\left\{\hat{A}(t, \boldsymbol{r}),\left\{\hat{A}\left(t, \boldsymbol{r}^{\prime}\right), \hat{\rho}\right\}\right\}\right)
$$

where

$$
\begin{aligned}
b_{R R}\left(t, \boldsymbol{r}, \boldsymbol{r}^{\prime}\right) & :=\frac{1}{4} \int d \boldsymbol{r}^{\prime \prime} \boldsymbol{r}^{\prime \prime \prime} \lambda_{R}\left(t, \boldsymbol{r}, \boldsymbol{r}^{\prime \prime}\right) \gamma^{-1}\left(t, \boldsymbol{r}^{\prime \prime}, \boldsymbol{r}^{\prime \prime \prime}\right) \lambda_{R}\left(t, \boldsymbol{r}^{\prime}, \boldsymbol{r}^{\prime \prime \prime}\right), \\
b_{I R}\left(t, \boldsymbol{r}, \boldsymbol{r}^{\prime}\right) & :=\frac{1}{4} \int d \boldsymbol{r}^{\prime \prime} \boldsymbol{r}^{\prime \prime \prime} \lambda_{I}\left(t, \boldsymbol{r}, \boldsymbol{r}^{\prime \prime}\right) \gamma^{-1}\left(t, \boldsymbol{r}^{\prime \prime}, \boldsymbol{r}^{\prime \prime \prime}\right) \lambda_{R}\left(t, \boldsymbol{r}^{\prime}, \boldsymbol{r}^{\prime \prime \prime}\right), \\
b_{I I}\left(t, \boldsymbol{r}, \boldsymbol{r}^{\prime}\right) & :=\frac{1}{4} \int d \boldsymbol{r}^{\prime \prime} \boldsymbol{r}^{\prime \prime \prime} \lambda_{I}\left(t, \boldsymbol{r}, \boldsymbol{r}^{\prime \prime}\right) \gamma^{-1}\left(t, \boldsymbol{r}^{\prime \prime}, \boldsymbol{r}^{\prime \prime \prime}\right) \lambda_{I}\left(t, \boldsymbol{r}^{\prime}, \boldsymbol{r}^{\prime \prime \prime}\right),
\end{aligned}
$$

and we have used $\left[\hat{A}^{2}, \hat{\rho}\right] \equiv\{\hat{A},[\hat{A}, \hat{\rho}]\} \equiv[\hat{A},\{\hat{A}, \hat{\rho}\}]$. Therefore, $\hat{\rho}(t)$ obeys the following master equation:

$$
\frac{d \hat{\rho}(t)}{d t}=\int d \boldsymbol{r} d \boldsymbol{r}^{\prime}\left(2 i b_{I R}\left(t, \boldsymbol{r}, \boldsymbol{r}^{\prime}\right)\left[\hat{A}(t, \boldsymbol{r}) \hat{A}\left(t, \boldsymbol{r}^{\prime}\right), \hat{\rho}\right]-b_{R R}\left(t, \boldsymbol{r}, \boldsymbol{r}^{\prime}\right)\left[\hat{A}(t, \boldsymbol{r}),\left[\hat{A}\left(t, \boldsymbol{r}^{\prime}\right), \hat{\rho}\right]\right]+b_{I I}\left(t, \boldsymbol{r}, \boldsymbol{r}^{\prime}\right)\left\{\hat{A}(t, \boldsymbol{r}),\left\{\hat{A}\left(t, \boldsymbol{r}^{\prime}\right), \hat{\rho}\right\}\right\}\right) .
$$

Finally, we take the nonrelativistic limit and replace $\hat{\phi}$ with the nonrelativistic scalar field $\hat{\Psi}$. The Hamiltonian density can then be thought of as in the form of that for a stochastic and complex generalization of the Newtonian gravitational interaction, with $h[\tilde{G}]$ a stochastic and complex generalization of the Newtonian potential and matter represented by $\hat{\Psi}$. Assuming that $\beta_{R R}, \beta_{I R}$, and $\beta_{R R}$ are time-independent, we end up with

$$
\begin{gathered}
\frac{d \hat{\rho}(t)}{d t}=\int d \boldsymbol{r} d \boldsymbol{r}^{\prime}\left(2 i b_{I R}\left(\boldsymbol{r}, \boldsymbol{r}^{\prime}\right)\left[\hat{\Psi}^{\dagger}(\boldsymbol{r}) \hat{\Psi}(\boldsymbol{r}) \hat{\Psi}^{\dagger}\left(\boldsymbol{r}^{\prime}\right) \hat{\Psi}\left(\boldsymbol{r}^{\prime}\right), \hat{\rho}(t)\right]-b_{R R}\left(\boldsymbol{r}, \boldsymbol{r}^{\prime}\right)\left[\hat{\Psi}^{\dagger}(\boldsymbol{r}) \hat{\Psi}(\boldsymbol{r}),\left[\hat{\Psi}^{\dagger}\left(\boldsymbol{r}^{\prime}\right) \hat{\Psi}\left(\boldsymbol{r}^{\prime}\right), \hat{\rho}(t)\right]\right]\right. \\
\left.+b_{I I}\left(\boldsymbol{r}, \boldsymbol{r}^{\prime}\right)\left\{\hat{\Psi}^{\dagger}(\boldsymbol{r}) \hat{\Psi}(\boldsymbol{r}),\left\{\hat{\Psi}^{\dagger}\left(\boldsymbol{r}^{\prime}\right) \hat{\Psi}\left(\boldsymbol{r}^{\prime}\right), \hat{\rho}(t)\right\}\right\}\right),
\end{gathered}
$$

where we have ignored the time dependence of $\hat{\Psi}$ for simplicity. The first term is of the same form as that which would be induced by the Newtonian limit of QG (see Appendix FA 2). However, despite Newtonian QG inducing non-Gaussianity (and negative Wigner functions), the other two terms conspire with the first to reduce the full process to a channel that keeps a Gaussian state in the (unnormalized) Gaussian convex hull. That is, despite the appearance of the first term, this master equation cannot turn a Gaussian state into a non-Gaussian state (defined as a state that lives outside the Gaussian convex hull). This is clear from our starting point (F10) for the averaged density matrix $\hat{\rho}$.

We can, however, write the solution of Eq. (F30) as a state in the standard quantum optics definition of the Gaussian convex hull by, for example, dropping the temporal and spatial dependence of $\tilde{\mathcal{G}}, \gamma, \lambda_{R}$, and $\lambda_{I}$ : the above master equation can then be written as 


$$
\begin{aligned}
\frac{d \hat{\rho}(t)}{d t}= & -i \kappa_{I R}\left[\left(\hat{a}^{\dagger} \hat{a}\right)^{2}, \hat{\rho}(t)\right]-\kappa_{R R}\left[\hat{a}^{\dagger} \hat{a},\left[\hat{a}^{\dagger} \hat{a}, \hat{\rho}(t)\right]\right] \\
& +\kappa_{I I}\left\{\hat{a}^{\dagger} \hat{a},\left\{\hat{a}^{\dagger} \hat{a}, \hat{\rho}(t)\right\}\right\},
\end{aligned}
$$

where we have also taken the single-mode approximation $\hat{\Psi}(\boldsymbol{r})=\psi(\boldsymbol{r}) \hat{a}$ found in the main text and defined $\kappa_{R R}:=$ $\frac{1}{4} \kappa^{2} \lambda_{R}^{2}, \kappa_{I R}:=\frac{1}{2} \kappa^{2} \lambda_{I} \lambda_{R}, \kappa_{I I}:=\frac{1}{4} \kappa^{2} \lambda_{I}^{2} ; \kappa:=\int d \boldsymbol{r}|\psi(\boldsymbol{r})|^{2}$, and $\gamma=\delta^{(3)}\left(\boldsymbol{r}-\boldsymbol{r}^{\prime}\right)$ for convenience. Using Eq. (F10),we can write the solution to Eq. (F31) as

$$
\hat{\rho}(t)=\int d g P(g, t) e^{-i \kappa \lambda_{R} \hat{a}^{\dagger} \hat{a} g t-\kappa \lambda_{I} \hat{a}^{\dagger} \hat{a} g t} \hat{\rho}(0) e^{i \kappa \lambda_{R} \hat{a}^{\dagger} \hat{a} g t-\kappa \lambda_{I} \hat{a}^{\dagger} \hat{a} g t},
$$

with

$$
P(g, t):=\sqrt{\frac{t}{\pi}} e^{-g^{2} t},
$$

where $g \in \mathbb{R}$ is a dummy variable used in place of $\tilde{\mathcal{G}}$. If $\hat{\rho}(0)$ in Eq. (F32) is a pure Gaussian state, the density matrix $\hat{\rho}(t)$ of Eq. (F32), which solves Eq. (F31), is then part of the (in general, non-normalized) Gaussian convex hull (F11).

\section{Preserving the norm: relationship to objective collapse theories and continuous-time measurements}

The master equations (F29) and (F30) [and so also Eq. (F31)] do not preserve the norm of the state. As detailed above, to preserve the norm, each stochastic density matrix can be redefined through Eq. (F8) and we can then take these as the physical stochastic density matrices. However, this results in a nonlinear evolution of the new averaged density matrix $\hat{\rho}$, which can lead to superluminal signaling $[179,182]$. This issue can also be found in objective-collapse theories where matter is coupled to a stochastic field through an anti-Hermitian term involving a particular matter operator $\hat{A}[180,181]$. In these models a term of the form $\hat{A}^{2}$ is included in the evolution of the stochastic state vector to eliminate the problematic nonlinear terms in the evolution of the averaged density matrix [180-182,249-253]. Such higher-order terms can also be used to eliminate the non-norm-preserving terms in the evolution of the non-normalized density matrix [182]. For example, to our Hamiltonian density (F12), we can add a term of the form $\hat{A}^{2}$ :

$$
\begin{aligned}
\hat{\mathcal{H}}[\tilde{\mathcal{G}}(x)]:= & \int d^{4} x^{\prime} \Lambda\left(x, x^{\prime}\right) \tilde{\mathcal{G}}\left(x^{\prime}\right) \hat{A}(x) \\
& -2 i \int d^{4} x^{\prime} \beta_{I I}\left(x, x^{\prime}\right) \hat{A}\left(x^{\prime}\right) \hat{A}(x),
\end{aligned}
$$

with $\hat{A}(x):=\hat{\phi}^{\dagger}(x) \hat{\phi}(x)$ and $\beta_{I I}$ defined in Eq. (F20). If we take the Markovian limit and assume a Gaussian profile for
$\tilde{\mathcal{G}}$ as above, the new term turns the non-norm-preserving term in Eq. (F29) into a norm-preserving term:

$$
\begin{aligned}
\frac{d \hat{\rho}(t)}{d t}= & \int d \boldsymbol{r} d \boldsymbol{r}^{\prime}\left\{2 i b_{I R}\left[\hat{A}(t, \boldsymbol{r}) \hat{A}\left(t, \boldsymbol{r}^{\prime}\right), \hat{\rho}(t)\right]\right. \\
& \left.-\left(b_{R R}+b_{I I}\right)\left[\hat{A}(t, \boldsymbol{r}),\left[\hat{A}\left(t, \boldsymbol{r}^{\prime}\right), \hat{\rho}(t)\right]\right]\right\} .
\end{aligned}
$$

Since $\hat{A}:=\hat{\phi}^{\dagger} \hat{\phi}$, the new term in Eq. (F34) is an (antiHermitian) quantum self-interaction of matter. That is, we have effectively introduced a new force. This new quantum force will, in general, induce non-Gaussianity. However, if we take $\Lambda_{R}=0$ in Eq. (F34) so that the stochastic interaction is anti-Hermitian (as is usually the case in objective-collapse theories), then the master equation simplifies to

$$
\frac{d \hat{\rho}(t)}{d t}=-\int d \boldsymbol{r} d \boldsymbol{r}^{\prime} b_{I I}\left(t, \boldsymbol{r}, \boldsymbol{r}^{\prime}\right)\left[\hat{A}(t, \boldsymbol{r}),\left[\hat{A}\left(t, \boldsymbol{r}^{\prime}\right), \hat{\rho}(t)\right]\right],
$$

which is a master equation that preserves the Gaussian convex hull since such a master equation is also derived when we take $\Lambda_{I}=0$ in the original theory without the new quantum self-interaction force [see Eq. (F29) with $\left.b_{I R}=b_{I I}=0\right]$. When we take the nonrelativistic limit $\hat{\phi} \rightarrow \hat{\Psi}$, Eq. (F36) is of the form of the master equation found in objective-collapse theories such as those of continuous spontaneous localization (CSL) and Diósi and Penrose [251,252,254]. It is also the master equation of continuous-time measurements in the basis $\hat{A}$, such that we can essentially consider the stochastic field $\tilde{\mathcal{G}}$ and new quantum self-interaction force $\beta_{I I} \hat{A}^{2}$ working together to perform continuous measurements of matter (that preserve the Gaussian convex hull).

If, however, both $\Lambda_{R}$ and $\Lambda_{I}$ are nonzero (see, e.g., Refs. [180,182] for similar models), then, in general, the non-Gaussian character of the new quantum selfinteraction force $\beta_{I I} \hat{A}^{2}$ is preserved, and we have a channel that can induce non-Gaussianity. Even so, in the asymptotic limit, the state will become a state of the Gaussian convex hull rather than a non-Gaussian state.

When both $\Lambda_{R}$ and $\Lambda_{I}$ are nonzero (and we also have the new quantum self-interaction force $\beta_{I I} \hat{A}^{2}$ ), the theory is closely related to a continuous-time measurement being performed by the two interactions as above but now with a feedback mechanism [182]. Weak measurements with local feedback operations can also induce entanglement in the case of joint measurements $[255,256]$.

[1] A. Einstein, Näherungsweise Integration der Feldgleichungen der Gravitation, Sitzungsberichte der Königlich 
Preußischen Akademie der Wissenschaften (Publishing House of the Royal Academy of Sciences, Berlin), Seite 688-696. (1916).

[2] C. Isham, Structural issues in quantum gravity, grqc/9510063 (1995).

[3] C. Rovelli, Strings, loops and others: a critical survey of the present approaches to quantum gravity, gr-qc/9803024 (1998).

[4] C. Rovelli, Notes for a brief history of quantum gravity, gr-qc/0006061 (2000).

[5] D. Oriti, Approaches to Quantum Gravity: Toward a new Understanding of Space, Time and Matter (Cambridge University Press, Cambridge, 2009).

[6] S. Carlip, D.-W. Chiou, W.-T. Ni, and R. Woodard, Quantum gravity: A brief history of ideas and some prospects, Int. J. Mod. Phys. D 24, 1530028 (2015).

[7] R. Penrose, On the gravitization of quantum mechanics 1: Quantum state reduction, Found. Phys. 44, 557 (2014).

[8] M. B. Green, J. Schwarz, and E. Witten, Superstring Theory: Volume 1. Introduction, Cambridge Monographs on Mathematical Physics (Cambridge University Press, 1988).

[9] M. B. Green, J. Schwarz, and E. Witten, Superstring Theory: Volume 2. Loop Amplitudes, Anomalies and Phenomenology (Cambridge University Press, Cambridge, U.K., 2012).

[10] J. Polchinski, String Theory. Vol. 2: Superstring Theory and Beyond, Cambridge Monographs on Mathematical Physics (Cambridge University Press, 2007).

[11] J. Polchinski, String Theory. Vol. 1: An Introduction to the Bosonic String, Cambridge Monographs on Mathematical Physics (Cambridge University Press, 2007).

[12] C. Rovelli and L. Smolin, in International Conference on Gravitation and Cosmology, Goa, edited by B. R. Iyer, A. Kembhavi, J. V. Narlikar, C. V. Vishveshwara (Cambridge University Press, Cambridge, U.K., 1988), p. 14.

[13] C. Rovelli, Quantum Gravity (Cambridge University Press, Cambridge, 2004).

[14] T. Thiemann, Modern Canonical Quantum General Relativity (Cambridge University Press, Cambridge, 2008).

[15] L. Rosenfeld, On quantization of fields, Nucl. Phys. 40, 353 (1963).

[16] S. Carlip, Is quantum gravity necessary? Classical Quantum Gravity 25, 154010 (2008).

[17] C. Møller, Les théories relativistes de la gravitation: Actes du colloque international, CNRS (CNRS, France, 1962), p. 15.

[18] T. Kibble, Relativistic models of nonlinear quantum mechanics, Commun. Math. Phys. 64, 73 (1978).

[19] T. W. B. Kibble and S. Randjbar-Daemi, Non-linear coupling of quantum theory and classical gravity, J. Phys. A: Math. Gen. 13, 141 (1980).

[20] Rather than using laboratory settings, such as a particle accelerator, it may also be possible to probe general Planck length or energy scale effects of QG through cosmological and astrophysical studies [257]—see Sec. IV E for a brief overview. However, these lack the control of laboratory settings and, because of this, the predicted QG effects tend to also be explainable by classical theories of gravity [197]. There are also proposals for tests of, specific phenomenological models of QG, rather than fundamental theories of QG, that are designed to show possible Planck length or energy scale effects, such as violations of Lorentz invariance, at accessible levels in particular experiments [258].

[21] S. Hossenfelder, Lost in Math: How Beauty Leads Physics Astray (Basic Books, New York, United States, 2018).

[22] M. Christodoulou and C. Rovelli, On the possibility of laboratory evidence for quantum superposition of geometries, Phys. Lett. B 792, 64 (2019).

[23] M. Bahrami, A. Bassi, S. McMillen, M. Paternostro, and H. Ulbricht, Is Gravity Quantum? arXiv:1507.05733 (2015).

[24] R. Howl, L. Hackermüller, D. E. Bruschi, and I. Fuentes, Gravity in the quantum lab, Adv. Phys.: X 3, 1383184 (2018).

[25] D. Kafri and J. M. Taylor, A noise inequality for classical forces, arXiv:1311.4558 [quant-ph] (2013).

[26] M. Carlesso, A. Bassi, M. Paternostro, and H. Ulbricht, Testing the gravitational field generated by a quantum superposition, New J. Phys. 21, 093052 (2019).

[27] S. Bose, A. Mazumdar, G. W. Morley, H. Ulbricht, M. Toroš, M. Paternostro, A. A. Geraci, P. F. Barker, M. S. Kim, and G. Milburn, Spin Entanglement Witness for Quantum Gravity, Phys. Rev. Lett. 119, 240401 (2017).

[28] R. J. Marshman, A. Mazumdar, and S. Bose, Locality \& Entanglement in Table-Top Testing of the Quantum Nature of Linearized Gravity, Phys. Rev. A 101, 052110 (2020).

[29] C. Marletto and V. Vedral, Gravitationally Induced Entanglement between two Massive Particles is Sufficient Evidence of Quantum Effects in Gravity, Phys. Rev. Lett. 119, 240402 (2017).

[30] C. Marletto and V. Vedral, When can gravity pathentangle two spatially superposed masses? Phys. Rev. D 98, 046001 (2018).

[31] T. Krisnanda, M. Zuppardo, M. Paternostro, and T. Paterek, Revealing Nonclassicality of Inaccessible Objects, Phys. Rev. Lett. 119, 120402 (2017).

[32] D. Kafri, J. M. Taylor, and G. J. Milburn, A classical channel model for gravitational decoherence, New J. Phys. 16, 065020 (2014).

[33] T. Krisnanda, G. Y. Tham, M. Paternostro, and T. Paterek, Observable quantum entanglement due to gravity, npj Quantum Inf. 6, 1 (2020).

[34] C. Wan, M. Scala, G. W. Morley, A. T. M. A. Rahman, H. Ulbricht, J. Bateman, P. F. Barker, S. Bose, and M. S. Kim, Free Nano-Object Ramsey Interferometry for Large Quantum Superpositions, Phys. Rev. Lett. 117, 143003 (2016).

[35] H. Pino, J. Prat-Camps, K. Sinha, B. P. Venkatesh, and O. Romero-Isart, On-chip quantum interference of a superconducting microsphere, Quantum Sci. Technol. 3, 025001 (2018).

[36] L. Parker, Particle Creation in Expanding Universes, Phys. Rev. Lett. 21, 562 (1968).

[37] L. Parker, Quantized fields and particle creation in expanding universes. I, Phys. Rev. 183, 1057 (1969). 
[38] L. Parker, Quantized fields and particle creation in expanding universes. II, Phys. Rev. D 3, 346 (1971).

[39] N. D. Birrell and P. C. W. Davies, Quantum Fields in Curved Space (Cambridge University Press, Cambridge, 1982).

[40] V. Vedral, Entanglement in the second quantization formalism, Open Phys. 1, 289 (2003).

[41] J. L. Ball, I. Fuentes-Schuller, and F. P. Schuller, Entanglement in an expanding spacetime, Phys. Lett. A 359, 550 (2006).

[42] I. Fuentes, R. B. Mann, E. Martín-Martínez, and S. Moradi, Entanglement of dirac fields in an expanding spacetime, Phys. Rev. D 82, 045030 (2010).

[43] S. Moradi, R. Pierini, and S. Mancini, Spin-particles entanglement in robertson-walker spacetime, Phys. Rev. D 89, 024022 (2014).

[44] M. J. W. Hall and M. Reginatto, On two recent proposals for witnessing nonclassical gravity, J. Phys. A: Math. Theor. 51, 085303 (2018).

[45] S. Pal, P. Batra, T. Paterek, and T. Mahesh, Experimental localisation of quantum entanglement through monitored classical mediator, arXiv:1909.11030 (2019).

[46] M. Reginatto and M. J. W. Hall, Entangling quantum fields via a classical gravitational interaction, J. Phys.: Conf. Ser. 1275, 012039 (2019).

[47] C. Marletto and V. Vedral, Answers to a few questions regarding the BMV experiment, arXiv:1907.08994 (2019).

[48] S. Lloyd and S. L. Braunstein, Quantum Computation Over Continuous Variables, Phys. Rev. Lett. 82, 1784 (1999).

[49] T. C. Ralph, A. Gilchrist, G. J. Milburn, W. J. Munro, and S. Glancy, Quantum computation with optical coherent states, Phys. Rev. A 68, 042319 (2003).

[50] R. D. Sorkin, Expressing entropy globally in terms of (4d) field-correlations, J. Phys.: Conf. Ser. 484, 012004 (2014).

[51] S. Floerchinger, Lectures on Quantum Fields and Information Theory (Heidelberg University, Heidelberg, Germany, 2018).

[52] J. Berges, S. Floerchinger, and R. Venugopalan, Dynamics of entanglement in expanding quantum fields, J. High Energy Phys. 2018, 145 (2018).

[53] B. V. Rajarama Bhat, T. C. John, and R. Srinivasan, Infinite mode quantum gaussian states, Rev. Math. Phys. 31, 1950030 (2019).

[54] C. Weedbrook, S. Pirandola, R. García-Patrón, N. J. Cerf, T. C. Ralph, J. H. Shapiro, and S. Lloyd, Gaussian quantum information, Rev. Mod. Phys. 84, 621 (2012).

[55] T. Kovachy, P. Asenbaum, C. Overstreet, C. Donnelly, S. Dickerson, A. Sugarbaker, J. Hogan, and M. Kasevich, Quantum superposition at the half-metre scale, Nature 528, 530 (2015).

[56] K. Lange, J. Peise, B. Lücke, I. Kruse, G. Vitagliano, I. Apellaniz, M. Kleinmann, G. Tóth, and C. Klempt, Entanglement between two spatially separated atomic modes, Science 360, 416 (2018).

[57] P. Kunkel, M. Prüfer, H. Strobel, D. Linnemann, A. Frölian, T. Gasenzer, M. Gärttner, and M. K. Oberthaler, Spatially distributed multipartite entanglement enables epr steering of atomic clouds, Science 360, 413 (2018).
[58] M. Fadel, T. Zibold, B. Décamps, and P. Treutlein, Spatial entanglement patterns and Einstein-Podolsky-Rosen steering in Bose-Einstein condensates, Science 360, 409 (2018).

[59] D. Linnemann, J. Schulz, W. Muessel, P. Kunkel, M. Prüfer, A. Frölian, H. Strobel, and M. K. Oberthaler, Active SU $(1,1)$ atom interferometry, Quantum Sci. Technol. 2, 044009 (2017).

[60] C. Chin, R. Grimm, P. Julienne, and E. Tiesinga, Feshbach resonances in ultracold gases, Rev. Mod. Phys. 82, 1225 (2010).

[61] L. Pitaevskii and S. Stringari, Bose-Einstein Condensation and Superfluidity (Oxford University Press, Oxford, 2016).

[62] M. E. Peskin and D. V. Schroeder, An introduction to quantum field theory (1995).

[63] J. F. Corney and P. D. Drummond, Gaussian operator bases for correlated fermions, J. Phys. A: Math. Gen. 39, 269 (2005).

[64] T. Shi, E. Demler, and J. Ignacio Cirac, Variational study of fermionic and bosonic systems with non-Gaussian states: Theory and applications, Ann. Phys. 390, 245 (2018).

[65] D. Glavan and T. Prokopec, A pedestrian introduction to non-equilibrium qft (2017).

[66] E. Greplova, Ph.D. thesis, LMU München, 2013.

[67] E. Wigner, On the quantum correction for thermodynamic equilibrium, Phys. Rev. 40, 749 (1932).

[68] M. Hillery, R. F. O’Connel, M. O. Scully, and E. P. Wigner, Distribution functions in physics: Fundamentals, Phys. Rep. 106, 121 (1984).

[69] A. Kenfack and K. Yczkowski, Negativity of the wigner function as an indicator of non-classicality, J. Opt. B: Quantum Semiclassical Opt. 6, 396 (2004).

[70] For pure states, the divide between states with positive and negative Wigner functions exactly coincides with the divide between states with Gaussian and non-Gaussian Wigner functions. In contrast, for general mixed states, although only non-Gaussian states can have negative Wigner functions, it is also possible for a non-Gaussian state to have a positive Wigner function.

[71] R. Hudson, When is the wigner quasi-probability density non-negative? Rep. Math. Phys. 6, 249 (1974).

[72] S. D. Bartlett, B. C. Sanders, S. L. Braunstein, and K. Nemoto, Efficient Classical Simulation of Continuous Variable Quantum Information Processes, Phys. Rev. Lett. 88, 097904 (2002).

[73] J. Eisert, S. Scheel, and M. B. Plenio, Distilling Gaussian States with Gaussian Operations is Impossible, Phys. Rev. Lett. 89, 137903 (2002).

[74] J. Fiurášek, Gaussian Transformations and Distillation of Entangled Gaussian States, Phys. Rev. Lett. 89, 137904 (2002).

[75] G. Giedke and J. Ignacio Cirac, Characterization of gaussian operations and distillation of gaussian states, Phys. Rev. A 66, 032316 (2002).

[76] K. Banaszek and K. Wódkiewicz, Nonlocality of the Einstein-Podolsky-Rosen state in the wigner representation, Phys. Rev. A 58, 4345 (1998). 
[77] K. Banaszek and K. Wódkiewicz, Testing Quantum Nonlocality in Phase Space, Phys. Rev. Lett. 82, 2009 (1999).

[78] R. Filip and L. Mišta, Violation of bell's inequalities for a two-mode squeezed vacuum state in lossy transmission lines, Phys. Rev. A 66, 044309 (2002).

[79] Z.-B. Chen, J.-W. Pan, G. Hou, and Y.-D. Zhang, Maximal Violation of Bell's Inequalities for Continuous Variable Systems, Phys. Rev. Lett. 88, 040406 (2002).

[80] H. Nha and H. J. Carmichael, Proposed Test of Quantum Nonlocality for Continuous Variables, Phys. Rev. Lett. 93, 020401 (2004).

[81] C. Invernizzi, S. Olivares, M. G. A. Paris, and K. Banaszek, Effect of noise and enhancement of nonlocality in on/off photodetection, Phys. Rev. A 72, 042105 (2005).

[82] R. García-Patrón, J. Fiurášek, and N. J. Cerf, Loopholefree test of quantum nonlocality using high-efficiency homodyne detectors, Phys. Rev. A 71, 022105 (2005).

[83] A. Ferraro and M. G. A. Paris, Nonlocality of twoand three-mode continuous variable systems, J. Opt. B: Quantum Semiclassical Opt. 7, 174 (2005).

[84] M. G. Genoni, M. G. A. Paris, and K. Banaszek, Measure of the non-gaussian character of a quantum state, Phys. Rev. A 76, 042327 (2007).

[85] Q. Zhuang, P. W. Shor, and J. H. Shapiro, Resource theory of non-gaussian operations, Phys. Rev. A 97, 052317 (2018).

[86] M. G. Genoni, M. G. A. Paris, and K. Banaszek, Quantifying the non-gaussian character of a quantum state by quantum relative entropy, Phys. Rev. A 78, 060303(R) (2008).

[87] F. Albarelli, M. G. Genoni, M. G. A. Paris, and A. Ferraro, Resource theory of quantum non-gaussianity and wigner negativity, Phys. Rev. A 98, 052350 (2018).

[88] M. G. Genoni and M. G. A. Paris, Quantifying nongaussianity for quantum information, Phys. Rev. A 82, 052341 (2010).

[89] P. Marian and T. A. Marian, Relative entropy is an exact measure of non-gaussianity, Phys. Rev. A 88, 012322 (2013).

[90] B. Dubost, M. Koschorreck, M. Napolitano, N. Behbood, R. J. Sewell, and M. W. Mitchell, Efficient Quantification of Non-Gaussian Spin Distributions, Phys. Rev. Lett. 108, 183602 (2012).

[91] M. K. Olsen and J. F. Corney, Non-gaussian continuousvariable entanglement and steering, Phys. Rev. A 87, 033839 (2013).

[92] C. Hughes, M. G. Genoni, T. Tufarelli, M. G. A. Paris, and M. S. Kim, Quantum non-gaussianity witnesses in phase space, Phys. Rev. A 90, 013810 (2014).

[93] M. G. Genoni, M. L. Palma, T. Tufarelli, S. Olivares, M. S. Kim, and M. G. A. Paris, Detecting quantum nongaussianity via the wigner function, Phys. Rev. A 87, 062104 (2013).

[94] B. L. Schumaker, Quantum mechanical pure states with gaussian wave functions, Phys. Rep. 135, 317 (1986).

[95] C. M. Cheng and P. C. W. Fung, The evolution operator technique in solving the schrodinger equation, and its application to disentangling exponential operators and solving the problem of a mass-varying harmonic oscillator, J. Phys. A: Math. Gen. 21, 4115 (1988).

[96] R. G. Littlejohn, The semiclassical evolution of wave packets, Phys. Rep. 138, 193 (1986).

[97] B. R. Mollow, Quantum statistics of coupled oscillator systems, Phys. Rev. 162, 1256 (1967).

[98] J. C. Sandoval-Santana, V. G. Ibarra-Sierra, J. L. Cardoso, and A. Kunold, Time evolution of two-dimensional quadratic hamiltonians: A lie algebraic approach, J. Math. Phys. 57, 042104 (2016).

[99] We do not include terms that are neither linear nor quadratic in $\hat{\phi}$, such as $\hat{\phi}^{3} \mathcal{G}$, since we consider these as inducing quantum self-interactions of $\hat{\phi}$. Later, when we consider $\mathcal{G}$ to be associated with gravitational interactions, such terms turn out to be excluded since we are working in a situation where all nongravitational interactions can be ignored. Similarly, if we single out any of the Standard Model forces and take them to be classical, then these terms are not present.

[100] By "matter," we mean the leptons and quarks of the Standard Model, although we could also include other quantum entities, such as the electromagnetic field. The leptons and quarks are described by spin-1/2 quantum fields, and we use a simple real scalar field $\hat{\phi}$ (for which particles are their own antiparticles) to describe matter only to illustrate our argument. The argument also applies to spin-1/2 fields.

[101] T. Thiemann, Quantum spin dynamics (QSD): V. quantum gravity as the natural regulator of the hamiltonian constraint of matter quantum field theories, Classical Quantum Gravity 15, 1281 (1998).

[102] C. Rovelli, Quantum Gravity, Cambridge Monographs on Mathematical Physics (Cambridge University Press, 2004).

[103] Also see Appendix A for a brief summary.

[104] R. Feynman, Quantum theory of gravitation, Acta Phys. Polon. 24, 697 (1963).

[105] B. S. DeWitt, Quantum theory of gravity. II. The manifestly covariant theory, Phys. Rev. 162, 1195 (1967).

[106] S. Gupta, Quantization of Einstein's gravitational field: General treatment, Proc. Phys. Soc. A 65, 608 (1952).

[107] See Appendix A 1 for the Hamiltonians of weak-field gravity.

[108] See Appendix A 2 for a derivation of Newtonian gravity from GR.

[109] By matter interactions, we mean all interactions of the quarks and leptons (i.e., the electroweak force, the strong force, and the gravitational force). We do not take, for example, the Higgs boson to be matter.

[110] B. Mielnik, Generalized quantum mechanics, Commun. Math. Phys. 37, 221 (1974).

[111] L. Diósi, Gravitation and quantum-mechanical localization of macro-objects, Phys. Lett. A 105, 199 (1984).

[112] R. Penrose, Quantum computation, entanglement and state reduction, Philos. Trans. R. Soc. London. Ser. A: Math., Phys. Eng. Sci. 356, 1927 (1998).

[113] R. Penrose, The Road to Reality: A Complete Guide to the Laws of the Universe (Jonathan Cape, London, 2004).

[114] The Schrödinger-Newton equations can refer to the CG theory where gravity is fundamentally sourced by the 
expectation value of quantum matter density [112,226], as well as to a limit of QG where a Hartree approximation for the mutual quantum gravitational interaction in a system of many particles is used. These two descriptions are fundamentally different, this being clearest in the singleparticle limit, where the latter description cannot be used but where the CG theory still applies and describes a single-particle wave function self-interacting gravitationally (which cannot happen in QG) [226]. Here by the Schrödinger-Newton equations, we refer to the fundamental CG theory rather than the QG approximation.

[115] Although $\lambda_{Q} G$ and $\lambda_{C} G$ may look very similar, they can take on very different values. For example, in the Schrödinger-Newton equations, $\lambda_{C} G=N \lambda_{Q} G$, where $N$ is the number of atoms of the BEC (see Appendix E).

[116] In the Newtonian limit, the QG Hamiltonian (8) appears as an effective quantum self-interaction of matter. In contrast, the CG Hamiltonian cannot induce quantum selfinteractions of matter [Eq. (9)]. This is because, in the absence of all other interactions, the CG Hamiltonian contains only the gravitational field coupled to the kinetic and mass terms of matter, which are quadratic in the matter field.

[117] J. F. Donoghue, Leading Quantum Correction to the Newtonian Potential, Phys. Rev. Lett. 72, 2996 (1994).

[118] J. F. Donoghue, General relativity as an effective field theory: The leading quantum corrections, Phys. Rev. D 50, 3874 (1994).

[119] A. Akhundov, S. Bellucci, and A. Shiekh, Gravitational interaction to one loop in effective quantum gravity, Phys. Lett. B 395, 16 (1997).

[120] N. E. J. Bjerrum-Bohr, J. F. Donoghue, and B. R. Holstein, Quantum gravitational corrections to the nonrelativistic scattering potential of two masses, Phys. Rev. D 67, 084033 (2003).

[121] C. P. Burgess, Quantum gravity in everyday life: General relativity as an effective field theory, Living Rev. Relativity 7, 5 (2004).

[122] S. A. Haine, Searching for Signatures of Quantum Gravity in Quantum Gases, arXiv:1810.10202 (2018).

[123] C. Gerry and P. L. Knight, Introductory Quantum Optics (Cambridge University Press, Cambridge, 2005).

[124] Entanglement has been observed in split BECs [56-58]. In these experiments the initial state was of two BECs in two hyperfine levels. Although the initial BECs do not appear to be entangled in the second-quantization picture, they do look entangled in the first-quantization picture (this is often referred to as "particle" entanglement). In contrast, since we have only a single BEC here in a single location, there is no entanglement in either quantization picture. In the second-quantization picture, a single ket is used to describe the system, such as $|\alpha\rangle$ for a coherent state. In the first-quantization picture, in the limit of absolute zero, and fixing the particle number as $N$, the state of the system is described by the many-body wave function $\Phi\left(\mathbf{r}_{1}, \mathbf{r}_{2}, \ldots \mathbf{r}_{N}\right)=\psi_{0}\left(\mathbf{r}_{1}\right) \psi_{0}\left(\mathbf{r}_{2}\right) \cdots \psi_{0}\left(\mathbf{r}_{N}\right)$, where $\psi_{0}$ are the identical wave functions for each atom.

[125] As indicated in Eq. (11), CG theories can be nonlinear in the evolution of the state vector [110]. However, even if it is nonlinear, it is still a Gaussian process since a CG theory must be quadratic in matter fields: the nonlinearity means that although we know a Gaussian state will remain a Gaussian state, we may not be able to analytically determine the specific evolution (see Appendix E for more details).

[126] M. G. Kendall and A. Stuart, The Advanced Theory of Statistics (Charles Griffin, London, 1958), Vol. 1.

[127] See Appendix D for the standard deviation of $k_{4}$.

[128] V. Giovannetti, S. Lloyd, and L. Maccone, Advances in quantum metrology, Nat. Photonics 5, 222 (2011).

[129] B. C. Sanders, Quantum dynamics of the nonlinear rotator and the effects of continual spin measurement, Phys. Rev. A 40, 2417 (1989).

[130] A. N. Boto, P. Kok, D. S. Abrams, S. L. Braunstein, C. P. Williams, and J. P. Dowling, Quantum Interferometric Optical Lithography: Exploiting Entanglement to Beat the Diffraction Limit, Phys. Rev. Lett. 85, 2733 (2000).

[131] S. Qvarfort, S. Bose, and A. Serafini, Mesoscopic entanglement from central potential interactions, J. Phys. B: Atomic, Molecular and Optical Phys. 53, 235501 (2020).

[132] R. Demkowicz-Dobrzaski, M. Jarzyna, and J. Koodyski, Chapter Four - Quantum Limits in Optical Interferometry (Elsevier, Amsterdam, Netherlands, 2015), p. 345.

[133] See Appendix D.

[134] R. Howl, R. Penrose, and I. Fuentes, Exploring the unification of quantum theory and general relativity with a Bose-Einstein condensate, New J. Phys. 21, 043047 (2019).

[135] D. G. Fried, T. C. Killian, L. Willmann, D. Landhuis, S. C. Moss, D. Kleppner, and T. J. Greytak, Bose-Einstein Condensation of Atomic Hydrogen, Phys. Rev. Lett. 81, 3811 (1998).

[136] K. M. R. van der Stam, E. D. van Ooijen, R. Meppelink, J. M. Vogels, and P. van der Straten, Large atom number Bose-Einstein condensate of sodium, Rev. Sci. Instrum. 78, 013102 (2007).

[137] C. K. Law, H. Pu, and N. P. Bigelow, Quantum Spins Mixing in Spinor Bose-Einstein Condensates, Phys. Rev. Lett. 81, 5257 (1998).

[138] R. J. Lewis-Swan and K. V. Kheruntsyan, Sensitivity to thermal noise of atomic Einstein-Podolsky-Rosen entanglement, Phys. Rev. A 87, 063635 (2013).

[139] S. S. Szigeti, R. J. Lewis-Swan, and S. A. Haine, PumpedUp su(1, 1) Interferometry, Phys. Rev. Lett. 118, 150401 (2017).

[140] W. Muessel, H. Strobel, D. Linnemann, D. B. Hume, and M. K. Oberthaler, Scalable Spin Squeezing for QuantumEnhanced Magnetometry with Bose-Einstein Condensates, Phys. Rev. Lett. 113, 103004 (2014).

[141] Alternatively, rather than using a Gaussian squeezed state, another highly nonclassical state could be used, such as a Schrödinger cat state consisting of a superposition of coherent states with different phases. For example, a Yurke-Stoler state [259] $(|\alpha\rangle+i|-\alpha\rangle) / \sqrt{2}$ could, in principle, be created in a BEC using a magnetic field to ramp up the electromagnetic interactions between the atoms before subsequently turning them off $[61,123]$. However, since the Yurke-Stoler state is non-Gaussian, we would have to either consider the change in the value of $\kappa_{4}$ or use a protocol where we evolve a single condensate in a coherent state $|\alpha\rangle$ to a Yurke-Stoler state, and then apply 
the reverse process such that the system returns to $|\alpha\rangle$ if the CG Hamiltonian (9) acts rather than QG.

[142] C. Kurtsiefer, T. Pfau, and J. Mlynek, Measurement of the wigner function of an ensemble of helium atoms, Nature 386, 150 (1997).

[143] C. Gross, H. Strobel, E. Nicklas, T. Zibold, N. Bar-Gill, G. Kurizki, and M. Oberthaler, Atomic homodyne detection of continuous-variable entangled twin-atom states, Nature 480, 219 (2011).

[144] S. Wüster, B. J. Dabrowska-Wüster, S. M. Scott, J. D. Close, and C. M. Savage, Quantum-field dynamics of expanding and contracting Bose-Einstein condensates, Phys. Rev. A 77, 023619 (2008).

[145] M. T. Johnsson and S. A. Haine, Generating Quadrature Squeezing in an Atom Laser through Self-Interaction, Phys. Rev. Lett. 99, 010401 (2007).

[146] S. S. Hodgman, R. G. Dall, A. G. Manning, K. G. H. Baldwin, and A. G. Truscott, Direct measurement of longrange third-order coherence in Bose-Einstein condensates, Science 331, 1046 (2011).

[147] M. F. Riedel, P. Böhi, Y. Li, T. W. Hänsch, A. Sinatra, and P. Treutlein, Atom-chip-based generation of entanglement for quantum metrology, Nature 464, 1170 (2010).

[148] R. Schmied and P. Treutlein, Tomographic reconstruction of the wigner function on the bloch sphere, New J. Phys. 13, 065019 (2011).

[149] R. McConnell, H. Zhang, J. Hu, S. Ćuk, and V. Vuletić, Entanglement with negative wigner function of almost 3000 atoms heralded by one photon, Nature 519, 439 (2015).

[150] R. McConnell, H. Zhang, J. Hu, S. Cuk, and V. Vuletić, Entanglement with negative wigner function of three thousand atoms heralded by one photon, J. Phys.: Conf. Ser. 723, 012054 (2016).

[151] J. S. Lundeen, B. Sutherland, A. Patel, C. Stewart, and C. Bamber, Direct measurement of the quantum wavefunction, Nature 474, 188 (2011).

[152] J. S. Lundeen and C. Bamber, Procedure for Direct Measurement of General Quantum States Using Weak Measurement, Phys. Rev. Lett. 108, 070402 (2012).

[153] D. Das Arvind, Weak measurement-based state estimation of gaussian states of one-variable quantum systems, J. Phys. A: Math. Theor. 50, 145307 (2017).

[154] G. S. Thekkadath, F. Hufnagel, and J. S. Lundeen, Determining complementary properties using weakmeasurement: Uncertainty, predictability, and disturbance, New J. Phys. 20, 113034 (2018).

[155] O. Hosten and P. Kwiat, Observation of the spin hall effect of light via weak measurements, Science 319, 787 (2008).

[156] M. Malik, M. Mirhosseini, M. P. Lavery, J. Leach, M. J. Padgett, and R. W. Boyd, Direct measurement of a 27dimensional orbital-angular-momentum state vector, Nat. Commun. 5, 3115 (2014).

[157] J. Z. Salvail, M. Agnew, A. S. Johnson, E. Bolduc, J. Leach, and R. W. Boyd, Full characterization of polarization states of light via direct measurement, Nat. Photonics 7, 316 (2013).

[158] C. Bamber and J. S. Lundeen, Observing Dirac's Classical Phase Space Analog to the Quantum State, Phys. Rev. Lett. 112, 070405 (2014).
[159] S. E. Pollack, D. Dries, M. Junker, Y. P. Chen, T. A. Corcovilos, and R. G. Hulet, Extreme Tunability of Interactions in a ${ }^{7} \mathrm{Li}$ Bose-Einstein Condensate, Phys. Rev. Lett. 102, 090402 (2009).

[160] Y. Huang, Y. Zhang, R. Lü, X. Wang, and S. Yi, Macroscopic quantum coherence in spinor condensates confined in an anisotropic potential, Phys. Rev. A 86, 043625 (2012).

[161] A. J. Olson, D. L. Whitenack, and Y. P. Chen, Effects of magnetic dipole-dipole interactions in atomic BoseEinstein condensates with tunable $s$-wave interactions, Phys. Rev. A 88, 043609 (2013).

[162] Q.-S. Tan, Q.-T. Xie, and L.-M. Kuang, Effects of dipolar interactions on the sensitivity of nonlinear spinor-bec interterometry, Sci. Rep. 8, 1 (2018).

[163] M. Fattori, G. Roati, B. Deissler, C. D’Errico, M. Zaccanti, M. Jona-Lasinio, L. Santos, M. Inguscio, and G. Modugno, Magnetic Dipolar Interaction in a Bose-Einstein Condensate Atomic Interferometer, Phys. Rev. Lett. 101, 190405 (2008).

[164] Rather than working with a spherical BEC, where MDDIs cancel, we could alternatively use a harmonic trap to create a quasi-one-dimensional or quasi-two-dimensional geometry (extreme prolate or oblate spheroidal geometries) [61]. In this case, instead of tuning the s-wave scattering length to zero, we can tune it to cancel the MDDIs such that we again have zero overall electromagnetic interactions [163]. We could also use pure two-dimensional or one-dimensional Bose gases where the trapping geometry has one or two dimensions with characteristic size much smaller than both two-body and three-body length scales [61], leading to a drastic reduction in collisional electromagnetic effects [61], but not gravitational effects [134]. In this case, there may be no phase transition to a BEC but there can still be macroscopic occupation of the ground state. Yet another option is to use ${ }^{88} \mathrm{Sr}$ rather than ${ }^{133} \mathrm{Cs}$. This has zero total magnetic moment, meaning that it has no MDDIs and is insensitive to external magnetic fields. It also has a very small s-wave scattering length [260], which is controllable through an optical Feshbach resonance [261,262].

[165] C. Chin, V. Vuletić, A. J. Kerman, S. Chu, E. Tiesinga, P. J. Leo, and C. J. Williams, Precision feshbach spectroscopy of ultracold cs $\mathrm{cs}_{2}$, Phys. Rev. A 70, 032701 (2004).

[166] A. D. Lange, K. Pilch, A. Prantner, F. Ferlaino, B. Engeser, H.-C. Nägerl, R. Grimm, and C. Chin, Determination of atomic scattering lengths from measurements of molecular binding energies near feshbach resonances, Phys. Rev. A 79, 013622 (2009).

[167] Rather than eliminating the noise from the electromagnetic interactions, we could use distinguishing features between the electromagnetic and gravitational interactions, such as how their strengths vary with the trapping potential and the magnitude of an applied magnetic field. For example, for a spherical trap, the effective strength of the electromagnetic interaction scales as $1 / R^{3}$, whereas gravity scales as $1 / R$ - see Eqs. (21) and (15). The different scaling is because the electromagnetic interactions are already partially screened due to the atoms having overall neutral charge, whereas the coupling of 
gravity is universal and so cannot be screened. With an applied magnetic field, it is even possible to change the swave interactions from being repulsive to attractive and vice versa, which is clearly impossible to achieve with gravity.

[168] M. Christodoulou and C. Rovelli, On the possibility of experimental detection of the discreteness of time, Frontiers Phys. 8, 207 (2020).

[169] B. Dalton and S. Ghanbari, Two mode theory of BoseEinstein condensates: Interferometry and the josephson model, J. Mod. Opt. 59, 287 (2012).

[170] N. Killoran, M. Cramer, and M. B. Plenio, Extracting Entanglement from Identical Particles, Phys. Rev. Lett. 112, 150501 (2014).

[171] E.-M. Graefe and R. Schubert, Wave-packet evolution in non-hermitian quantum systems, Phys. Rev. A 83, 060101(R) (2011).

[172] E.-M. Graefe and R. Schubert, Complexified coherent states and quantum evolution with non-hermitian hamiltonians, J. Phys. A: Math. Theor. 45, 244033 (2012).

[173] E.-M. Graefe, H. J. Korsch, A. Rush, and R. Schubert, Classical and quantum dynamics in the (non-hermitian) swanson oscillator, J. Phys. A: Math. Theor. 48, 055301 (2015).

[174] In general, the matter state will no longer be normalized, and by the Gaussian "form" of a Wigner function we mean a Wigner function that is a general Gaussian function, which then becomes a Gaussian probability density if the state is normalized [171-173]. The physical density matrix can be defined as $|\Psi\rangle\langle\Psi| /\langle\Psi \mid \Psi\rangle$, such that the final state would, in principle, be automatically normalized. However, it is possible that $\langle\Psi \mid \Psi\rangle$ is ill-defined, and here we assume that any such unphysical states that may result from the action of the non-Hermitian Hamiltonian are forbidden, perhaps due to an additional constraint on the interaction [171-173].

[175] J. Oppenheim, A post-quantum theory of classical gravity? arXiv:1811.03116 (2018).

[176] A Gaussian completely positive map is defined to be a completely positive map that takes any state with a Gaussian Wigner function to a state with a Gaussian Wigner function. The Clifford group [72] is defined to be the group of Bogoliubov transformations, whereas the Clifford semigroup [263] is the set of Gaussian completely positive maps. As with Bogoliubov transformations, the Clifford semigroup involves linear transformations of the quadratures, but the commutation relations do not have to be preserved. In Ref. [175], a Lindblad master equation with linear (matter) Lindblad operators is obtained for the interaction of matter with gravity, which is known to be a Gaussian completely positive map [264,265].

[177] T. Bröcker and R. F. Werner, Mixed states with positive wigner functions, J. Math. Phys. 36, 62 (1995).

[178] See Appendix F for more details.

[179] N. Gisin, Stochastic quantum dynamics and relativity, Helv. Phys. Acta 62, 363 (1989).

[180] G. Gasbarri, M. Toroš, S. Donadi, and A. Bassi, Gravity induced wave function collapse, Phys. Rev. D 96, 104013 (2017).
[181] S. L. Adler, Quantum Theory as an Emergent Phenomenon: The Statistical Mechanics of Matrix Models as the Precursor of Quantum Field Theory (Cambridge University Press, Cambridge, 2004).

[182] L. Diósi, Relativistic theory for continuous measurement of quantum fields, Phys. Rev. A 42, 5086 (1990).

[183] L. Diósi, Is spontaneous wave function collapse testable at all? J. Phys.: Conf. Ser. 626, 012008 (2015).

[184] It is not possible for the interaction with the stochastic entity $\mathcal{G}$ to perform continuous-time measurements of matter alone: a higher-order process, as discussed in the main text, must also be introduced (see Appendix F and, e.g., Refs. [182,266] for more details).

[185] As illustrated in Appendix F, even when the real and the imaginary components of $\mathcal{G}$ are both stochastic and obey a Gaussian probability distribution functional, the state of matter will still asymptotically tend to a Gaussian state, despite the presence of the new quantum self-interaction force. Of course, since objective-collapse theories are introduced in an attempt to explain the so-called measurement problem of QT (see, e.g., Refs. [32,180-182,245, 250,252,266-271] for gravitationally inspired theories), the state of matter must always tend to a classical-like state in these theories. When the real and imaginary components are both stochastic and there is a process related to quantum self-interaction, then the resulting theory is closely related to a continuous-time measurement being performed by the two interactions but now with a feedback mechanism [182]. Weak measurements with local feedback operations are also known to induce entanglement if the measurement is a joint measurement [255,256].

[186] T. Clifton, P. G. Ferreira, A. Padilla, and C. Skordis, Modified gravity and cosmology, Phys. Rep. 513, 1 (2012), modified Gravity and Cosmology.

[187] H. A. Buchdahl, Non-linear lagrangians and cosmological theory, Mon. Not. R. Astron. Soc. 150, 1 (1970).

[188] D. Baumann, The Physics of Inflation (CreateSpace Independent Publishing Platform, Scotts Valley, California, 2015).

[189] J. Martin, in Planck Scale Effects in Astrophysics and Cosmology, edited by Kowalski-Glikman, Jurek and Amelino-Camelia, Giovanni (Springer, Berlin Heidelberg, 2005), p. 199.

[190] This is a gauge-dependent statement since we can always choose the unitary gauge where quantum fluctuations of the inflaton vanish, and non-Gaussianity of curvature perturbations is then due entirely to nonlinear QG effects [188].

[191] R. K. Sachs and A. M. Wolfe, Perturbations of a cosmological model and angular variations of the microwave background, Astrophys. J. 147, 73 (1967).

[192] T. Battefeld and R. Easther, Non-gaussianities in multifield inflation, J. Cosmol. Astroparticle Phys. 2007, 020 (2007).

[193] A. P. Yadav and B. D. Wandelt, Primordial nongaussianity in the cosmic microwave background, Adv. Astron. 2010, 1 (2010).

[194] N. Bartolo, S. Matarrese, and A. Riotto, Cosmic microwave background anisotropies at second order: I, J. Cosmol. Astroparticle Phys. 2006, 024 (2006). 
[195] L. M. Krauss and F. Wilczek, Using cosmology to establish the quantization of gravity, Phys. Rev. D 89, 047501 (2014).

[196] J. Martin and V. Vennin, Obstructions to bell cmb experiments, Phys. Rev. D 96, 063501 (2017).

[197] J. Martin and V. Vennin, Quantum discord of cosmic inflation: Can we show that $\mathrm{cmb}$ anisotropies are of quantum-mechanical origin? Phys. Rev. D 93, 023505 (2016).

[198] J. Maldacena, A model with cosmological bell inequalities, Fortschritte Phys. 64, 10 (2016).

[199] P.-H. Chavanis, Statistical mechanics of self-gravitating systems in general relativity: I. The quantum fermi gas, Eur. Phys. J. Plus 135, 1 (2020).

[200] P.-H. Chavanis, Statistical mechanics of self-gravitating systems in general relativity: I. The quantum fermi gas, Eur. Phys. J. Plus 135, 1 (2020).

[201] R. C. Tolman, On the weight of heat and thermal equilibrium in general relativity, Phys. Rev. 35, 904 (1930).

[202] R. C. Tolman and P. Ehrenfest, Temperature equilibrium in a static gravitational field, Phys. Rev. 36, 1791 (1930).

[203] D. Oriti, in Quantum Gravity: Mathematical Models and Experimental Bounds, edited by B. Fauser, J. Tolksdorf, and E. Zeidler (Birkhäuser Basel, Basel, 2007), p. 101.

[204] L. Freidel, Group field theory: An overview, Int. J. Theor. Phys. 44, 1769 (2005).

[205] S. Weinberg, in Understanding the Fundamental Constituents of Matter, edited by Zichichi, Antonino (Springer, Boston, MA, 1978), p. 1.

[206] S. Weinberg, in General Relativity (1979).

[207] C. Burgess, An introduction to effective field theory, Annu. Rev. Nucl. Part. Sci. 57, 329 (2007).

[208] H. Georgi, Effective field theory, Annu. Rev. Nucl. Part. Sci. 43, 209 (1993).

[209] A. Peres and D. R. Terno, Quantum information and relativity theory, Rev. Mod. Phys. 76, 93 (2004).

[210] W. K. Wootters, A wigner-function formulation of finitestate quantum mechanics, Ann. Phys. 176, 1 (1987).

[211] A. Vourdas, Quantum systems with finite hilbert space, Rep Prog. Phys. 67, 267 (2004).

[212] C. Miquel, J. P. Paz, and M. Saraceno, Quantum computers in phase space, Phys. Rev. A 65, 062309 (2002).

[213] K. S. Gibbons, M. J. Hoffman, and W. K. Wootters, Discrete phase space based on finite fields, Phys. Rev. A 70, 062101 (2004).

[214] K. Bongs, M. Holynski, J. Vovrosh, P. Bouyer, G. Condon, E. Rasel, C. Schubert, W. P. Schleich, and A. Roura, Taking atom interferometric quantum sensors from the laboratory to real-world applications, Nat. Rev. Phys. 1, 731 (2019).

[215] F. J. Belinfante, On the covariant derivative of tensorundors, Physica 7, 305 (1940).

[216] M. M. Hatalkar, Theory of elementary particles in general relativity, Phys. Rev. 94, 1472 (1954).

[217] R. Penrose, Zero rest-mass fields including gravitation: Asymptotic behaviour, Proc. R. Soc. Lond. A. Math. Phys. Sci. 284, 159 (1965).
[218] J. S. Dowker, Y. P. Dowker, and B. H. Flowers, Interactions of massless particles of arbitrary spin, Proc. R. Soc. Lond. A. Math. Phys. Sci. 294, 175 (1966).

[219] J. S. Dowker and Y. P. Dowker, Particles of arbitrary spin in curved spaces, Proc. Phys. Soc. 87, 65 (1966).

[220] J. Dowker, Propagators for arbitrary spin in an einstein universe, Ann. Phys. 71, 577 (1972).

[221] G. Grensing, Quantised fields over de sitter space, J. Phys. A: Math. Gen. 10, 1687 (1977).

[222] S. Christensen and M. Duff, Axial and conformal anomalies for arbitrary spin in gravity and supergravity, Phys. Lett. B 76, 571 (1978)

[223] N. D. Birrell, Stress tensor conformal anomaly for weinberg-type fields in curved spacetimes, J. Phys. A: Math. Gen. 12, 337 (1979).

[224] M. Maggiore, Gravitational Waves: Volume 1: Theory and Experiments (Oxford University Press, Oxford, 2008).

[225] Even in the Newtonian approximation it is still possible for a quantum system to have an indefinite particle number (i.e. to not be an eigenstate of the number operator for the system). For example, the system could be an open quantum system.

[226] D. Carney, P. C. E. Stamp, and J. M. Taylor, Tabletop experiments for quantum gravity: A user's manual, Classical Quantum Gravity 36, 034001 (2019).

[227] M. Bahrami, A. Großardt, S. Donadi, and A. Bassi, The schrödinger-newton equation and its foundations, New J. Phys. 16, 115007 (2014).

[228] A. Großardt, J. Bateman, H. Ulbricht, and A. Bassi, Effects of newtonian gravitational self-interaction in harmonically trapped quantum systems, Sci. Rep. 6, 1 (2016).

[229] C. L. Benavides-Riveros and J. M. Gracia-Bondía, Physical wigner functions, Phys. Rev. A 87, 022118 (2013).

[230] K. Eckert, J. Schliemann, D. Bruß, and M. Lewenstein, Quantum correlations in systems of indistinguishable particles, Ann. Phys. 299, 88 (2002).

[231] G. Ghirardi, L. Marinatto, and T. Weber, Entanglement and properties of composite quantum systems: A conceptual and mathematical analysis, J. Stat. Phys. 108, 49 (2002).

[232] G. C. Ghirardi and L. Marinatto, General criterion for the entanglement of two indistinguishable particles, Phys. Rev. A 70, 012109 (2004).

[233] M. C. Tichy, F. Mintert, and A. Buchleitner, Essential entanglement for atomic and molecular physics, J. Phys. B: At., Mol. Opt. Phys. 44, 192001 (2011).

[234] M. Tichy, F. de Melo, M. Kus, F. Mintert, and A. Buchleitner, Entanglement of identical particles and the detection process, Fortschritte Phys. 61, 225 (2013).

[235] B. Morris, B. Yadin, M. Fadel, T. Zibold, P. Treutlein, and G. Adesso, Entanglement between identical particles is a useful and consistent resource, Phys. Rev. X 10, 041012 (2019).

[236] W. S. Bakr, J. I. Gillen, A. Peng, S. Fölling, and M. Greiner, A quantum gas microscope for detecting single atoms in a hubbard-regime optical lattice, Nature 462, 74 (2009). 
[237] W. S. Bakr, A. Peng, M. E. Tai, R. Ma, J. Simon, J. I. Gillen, S. Fölling, L. Pollet, and M. Greiner, Probing the superfluid-to-mott insulator transition at the single-atom level, Science 329, 547 (2010).

[238] J. F. Sherson, C. Weitenberg, M. Endres, M. Cheneau, I. Bloch, and S. Kuhr, Single-atom-resolved fluorescence imaging of an atomic mott insulator, Nature 467, 68 (2010).

[239] A. Perrin, R. Bücker, S. Manz, T. Betz, C. Koller, T. Plisson, T. Schumm, and J. Schmiedmayer, Hanbury brown and twiss correlations across the bose-einstein condensation threshold, Nat. Phys. 8, 195 (2012).

[240] F. Brennecke, R. Mottl, K. Baumann, R. Landig, T. Donner, and T. Esslinger, Real-time observation of fluctuations at the driven-dissipative dicke phase transition, Proc. Natl. Acad. Sci. 110, 11763 (2013).

[241] H. Ritsch, P. Domokos, F. Brennecke, and T. Esslinger, Cold atoms in cavity-generated dynamical optical potentials, Rev. Mod. Phys. 85, 553 (2013).

[242] F. Haas, J. Volz, R. Gehr, J. Reichel, and J. Estève, Entangled states of more than 40 atoms in an optical fiber cavity, Science 344, 180 (2014).

[243] S. Barnett and P. M. Radmore, Methods in Theoretical Quantum Optics (Oxford University Press, Oxford, 2002), Vol. 15.

[244] H.-P. Breuer and F. Petruccione, The Theory of Open Qunatum Systems (Oxford University Press, Oxford, 2002), 2nd ed.

[245] L. Diósi, Orthogonal jumps of the wavefunction in white-noise potentials, Phys. Lett. A 112, 288 (1985).

[246] N. Gisin, Quantum Measurements and Stochastic Processes, Phys. Rev. Lett. 52, 1657 (1984).

[247] A state in the Gaussian convex hull can have an overall non-Gaussian Wigner function.

[248] Here we are assuming, as in the main text, that the interaction is of the form of Eq. (F1) or, when extended to stochastic fields, Eq. (F2).

[249] G. C. Ghirardi, A. Rimini, and T. Weber, in Quantum Probability and Applications II, edited by L. Accardi and W. von Waldenfels (Springer, Berlin, Heidelberg, 1985), p. 223.

[250] G. C. Ghirardi, A. Rimini, and T. Weber, Unified dynamics for microscopic and macroscopic systems, Phys. Rev. D 34, 470 (1986).

[251] L. Diósi, Models for universal reduction of macroscopic quantum fluctuations, Phys. Rev. A 40, 1165 (1989)

[252] G. C. Ghirardi, P. Pearle, and A. Rimini, Markov processes in hilbert space and continuous spontaneous localization of systems of identical particles, Phys. Rev. A 42, 78 (1990).

[253] S. L. Adler, Comments on proposed gravitational modifications of schrödinger dynamics and their experimental implications, J. Phys. A: Math. Theor. 40, 755 (2007).

[254] If we take $\Lambda_{R}=0, \Lambda_{I}\left(x, x^{\prime}\right)=:-\sqrt{\lambda} \delta^{(4)}\left(x-x^{\prime}\right)$, and $\Gamma=\delta^{(4)}\left(x-x^{\prime}\right)$ and replace $\hat{A}$ with $(\hat{A}-\langle\hat{A}\rangle)$, the Hamiltonian density (F34) becomes $-i \hat{\mathcal{H}}[\tilde{\mathcal{G}}]=\sqrt{\lambda}(\hat{A}-$ $\langle\hat{A}\rangle) \tilde{\mathcal{G}}-\frac{1}{2} \lambda(\hat{A}-\langle\hat{A}\rangle)^{2}$, F37, which is closely connected to the evolution of the state vector found in objectivecollapse theories such as CSL and the Diósi-Penrose theory $[180,251,252]$.

[255] C. Hill and J. Ralph, Weak measurement and control of entanglement generation, Phys. Rev. A 77, 014305 (2008).

[256] L. Martin, F. Motzoi, H. Li, M. Sarovar, and K. B. Whaley, Deterministic generation of remote entanglement with active quantum feedback, Phys. Rev. A 92, 062321 (2015).

[257] A. Barrau, Testing different approaches to quantum gravity with cosmology: An overview, C. R. Phys. 18, 189 (2017).

[258] S. Hossenfelder and L. Smolin, Phenomenological quantum gravity, Phys. Can. 66, 99 (2010).

[259] B. Yurke and D. Stoler, Generating Quantum Mechanical Superpositions of Macroscopically Distinguishable States via Amplitude Dispersion, Phys. Rev. Lett. 57, 13 (1986).

[260] P. G. Mickelson, Y. N. Martinez, A. D. Saenz, S. B. Nagel, Y. C. Chen, T. C. Killian, P. Pellegrini, and R. Côté, Spectroscopic Determination of the $s$-Wave Scattering Lengths of ${ }^{86} \mathrm{Sr}$ and ${ }^{88} \mathrm{Sr}$, Phys. Rev. Lett. 95, 223002 (2005).

[261] P. G. Mickelson, Y. N. Martinez De Escobar, M. Yan, R. Chakraborty, and T. C. Killian, in 2009 Conference on Lasers and Electro-Optics and 2009 Conference on Quantum Electronics and Laser Science Conference (Optical Society of America, Washington, D.C., 2009), p. 1.

[262] S. Blatt, T. L. Nicholson, B. J. Bloom, J. R. Williams, J. W. Thomsen, P. S. Julienne, and J. Ye, Measurement of Optical Feshbach Resonances in an Ideal gas, Phys. Rev. Lett. 107, 073202 (2011).

[263] S. D. Bartlett and B. C. Sanders, Efficient Classical Simulation of Optical Quantum Information Circuits, Phys. Rev. Lett. 89, 207903 (2002).

[264] A. Serafini, M. G. A. Paris, F. Illuminati, and S. D. Siena, Quantifying decoherence in continuous variable systems, J. Opt. B: Quantum Semiclassical Opt. 7, R19 (2005).

[265] A. Ferraro, S. Olivares, and M. Paris, Gaussian States in Continuous Variable Quantum Information, Napoli Series on Physics and AstrophysicsBibliopolis, Napoli, 2005.

[266] K. Jacobs and D. A. Steck, A straightforward introduction to continuous quantum measurement, Contemp. Phys. 47, 279 (2006).

[267] L. Diósi, Continuous quantum measurement and itô formalism, Phys. Lett. A 129, 419 (1988).

[268] V. Belavkin, A new wave equation for a continuous nondemolition measurement, Phys. Lett. A 140, 355 (1989).

[269] N. Gisin and I. C. Percival, The quantum-state diffusion model applied to open systems, J. Phys. A: Math. Gen. 25, 5677 (1992).

[270] L. Diosi, Quantum stochastic processes as models for state vector reduction, J. Phys. A: Math. Gen. 21, 2885 (1988).

[271] E. Joos, H. D. Zeh, C. Kiefer, D. J. Giulini, J. Kupsch, and I.-O. Stamatescu, Decoherence and the Appearance of a Classical World in Quantum Theory (Springer Science, Berlin Heidelberg, 2013). 